\title{
How Persistent Are Shocks to World Commodity Prices?
}

\author{
PAUL CASHIN, HONG LIANG, and C. JOHN MCDERMOTT*
}

This paper examines the persistence of shocks to world commodity prices, using monthly IMF data on primary commodities between 1957-98. We find that shocks to commodity prices are typically long-lasting and the variability of the persistence of price shocks is quite wide. The paper also discusses the implications of these findings for national and international schemes to stabilize earnings from commodity exports and finds that if price shocks are long-lived, then the cost of stabilization schemes will likely exceed any associated smoothing benefits. [JEL C22, O13, O19, Q11, Q17]

his paper examines a key characteristic of the behavior of commodity pricesthe persistence of shocks to the prices of primary commodities. This is a topic of great importance, given that about 25 percent of world merchandise trade consists of primary commodities, and both long-term trends and short-term fluctuations in primary commodity prices are key determinants of developments in the world economy. On the supply side of the market, many developing countries continue to rely heavily on one or two primary commodities for the bulk of their export earnings, and primary commodities (including fuel and energy) constitute, on average, about half of export revenues of developing countries (see Table 1).

\footnotetext{
*Paul Cashin and Hong Liang are Economists in the IMF's Research Department. C. John McDermott is an Advisor at the Reserve Bank of New Zealand. The authors thank Will Martin, Catherine Pattillo, Mark Watson, two anonymous referees, seminar participants at the University of Melbourne, the Reserve Bank of New Zealand, the Australian Agricultural and Resource Economics Society, and the IMF for useful comments. The authors also gratefully acknowledge the extensive and helpful suggestions on earlier drafts of the paper provided by colleagues in the Commodities and Special Issues Division of the Research Department, particularly Peter Wickham, Blair Rourke, Miguel Savastano, Ximena Cheetham, and Thomas Tewksbury, and thank Ivan Guerra for excellent research assistance.
} 
On the demand side, commodity markets play a nontrivial role in transmitting business cycle disturbances and in affecting inflation rates in industrial nations (Borensztein and Reinhart, 1994). Our main goal in this paper is to estimate the duration of shocks to commodity prices. For commodity-dependent countries, knowledge of the duration of such shocks is an essential input to the design of policies to dampen the domestic economic effects of external shocks. In this paper we measure the persistence of shocks to commodity prices over the period 1957 to 1998 , using data on 60 indices of primary commodity prices.

An extensive literature exists on long-term trends in primary commodity prices. Classical economists suggested that the long-run trend of raw material prices was rising, because of limited supplies of natural resources in the face of diminishing returns to commodity production and growing populations. However, the Prebisch-Singer (1950) hypothesis argued that there was a declining long-term trend in primary commodity prices relative to manufactured goods, owing to the low income elasticity of demand for commodities and rapid increases in supply. The evidence in support of a persistent downward trend in relative commodity prices is rather mixed. While most scholars agree that relative commodity prices are nonstationary, they disagree as to whether the nonstationarity of prices takes the form of a deterministic trend, a stochastic trend, or whether there are structural breaks (Grilli and Yang, 1988; Cuddington and Urzúa, 1989; Reinhart and Wickham, 1994; and León and Soto, 1997).

For policy purposes, a good understanding of the cyclical behavior of commodity prices is equally as important as an understanding of their underlying long-run trends. ${ }^{1}$ In particular, reliable estimates of the duration of commodity price cycles are essential when considering counter-cyclical stabilization policies in primary-commodity exporting countries. Traditionally, however, the estimation of cycles is crucially dependent on whether the commodity price series are characterized as a unit root process. If the commodity price series are better modeled as a trend-stationary process, innovations in prices have no permanent effects. In such cases innovations are entirely cyclical, and commodity exporting countries can potentially benefit from price stabilization policies, such as the operation of a commodity stabilization scheme. On the other hand, if there is a unit root in the underlying data-generating process, shocks will have permanent effects, leaving very little room for any price stabilization scheme to work successfully. In contrast to this stark, and relatively uninformative, dichotomy between finite and permanent effects of innovations in commodity prices, in this paper we calculate the length of time typically taken for the effects of price shocks to dissipate.

The contributions of this paper are fourfold. First, we use the median-unbiased estimator proposed by Andrews (1993) to obtain an exact point and interval estimate of the autoregressive parameter in the commodity price data. This econometric procedure is superior to the results emanating from standard (least squares) unit root regressions, as least squares estimates are biased and any associated unit

\footnotetext{
${ }^{1}$ See Deaton and Laroque (1992) and Deaton (1992) for analyses of the time series characteristics of commodity prices. They find that commodity price cycles tend to be characterized by short-lived booms (typically triggered by low stock holdings), sharp busts, and long periods of flat prices.
} 


\section{Table 1. Countries Dependent on a Single Primary Commodity for Export Earnings}

(annual average of export data, U.S. dollars, 1992-97)

$\begin{array}{lll}\begin{array}{l}\text { For 50 Percent or More } \\ \text { of Export Earnings }\end{array} & \begin{array}{l}\text { For 20-49 Percent } \\ \text { of Export Earnings }\end{array} & \begin{array}{l}\text { For 10-19 Percent } \\ \text { of Export Earnings }\end{array}\end{array}$

\section{Countries in Middle East}

$\begin{array}{ll}\text { Crude petroleum } & \text { Bahrain } \\ & \text { Iran, Islamic Rep } \\ & \text { Iraq } \\ & \text { Kuwait } \\ & \text { Libya } \\ & \text { Oman } \\ \text { Qatar } \\ \text { Saudi Arabia } \\ \text { Yemen, Rep. }\end{array}$

Aluminum

Syrian Arab Rep.
United Arab Emirates

Egypt

Iraq

Kuwait

Saudi Arabia

Yemen, Rep.

\section{Countries in Africa}

Crude petroleum

\section{Angola \\ Congo, Rep. \\ Gabon \\ Nigeria}

Natural gas

Bauxite and alumina

Iron ore

Rutile

Copper

Cobalt

Gold

Diamonds

Uranium

Timber (African hardwood)

Cotton

Tobacco
Botswana

Niger

Ghana

South Africa

Central African Rep.

Namibia

Sierra Leone

Equatorial Guinea

Benin

Chad

Mali

Sudan

Zimbabwe

Malawi
Bahrain

Algeria

Equatorial Guinea

Algeria

Mauritania

Sierra Leone

Congo, Dem. Rep.

Congo, Dem. Rep. Zambia

Mali

Zimbabwe

Congo, Dem. Rep.

Central African Rep.

Gabon

Ghana

Swaziland

Burkina Faso 


\section{Table 1. (continued)}

$\begin{array}{lll}\begin{array}{l}\text { For 50 Percent or More } \\ \text { of Export Earnings }\end{array} & \begin{array}{l}\text { For 20-49 Percent } \\ \text { of Export Earnings }\end{array} & \begin{array}{l}\text { For 10-19 Percent } \\ \text { of Export Earnings }\end{array}\end{array}$

\section{Countries in Africa (continued)}

$\begin{array}{lll}\text { Arabica coffee } & \text { Burundi } & \text { Rwanda } \\ & \text { Ethiopia }\end{array}$

Robusta coffee Uganda

Cocoa São Tomé and Príncipe

Côte d'Ivoire

Cameroon

Ghana

Tea

Kenya

Rwanda

Vanilla

Comoros

Sugar

Mauritius

Swaziland

Cashew nuts

Guinea Bissau

Livestock

Mali

Niger

Sudan

Fish

Mauritania

Mozambique

Senegal

Namibia

Oilseeds

Sudan

\section{Countries in Western Hemisphere}

Crude petroleum Venezuela

Ecuador Colombia

Trinidad and Tobago

Mexico

Bauxite and

Jamaica

Guyana

alumina

Surinam

Copper

Chile

Peru

Gold

Guyana

Cotton

Arabica coffee

Guyana

St. Kitts and Nevis

Paraguay

Colombia

El Salvador

Guatemala

Honduras

Nicaragua

Sugar

St. Vincent

Belize

Bananas

Honduras

St. Lucia

Costa Rica

Livestock

Nicaragua

Fish

Ecuador

Fishmeal

Peru

Rice

Guyana 


\section{Table 1. (concluded)}

$\begin{array}{lll}\begin{array}{l}\text { For 50 Percent or More } \\ \text { of Export Earnings }\end{array} & \begin{array}{l}\text { For 20-49 Percent } \\ \text { of Export Earnings }\end{array} & \begin{array}{l}\text { For 10-19 Percent } \\ \text { of Export Earnings }\end{array}\end{array}$

Countries in Europe, Asia, and the Pacific

Crude petroleum

$\begin{array}{ll}\text { Azerbaijan } & \text { Indonesia } \\ \text { Brunei Darussalem } & \text { Kazakhstan } \\ \text { Norway } & \text { Vietnam } \\ \text { Papua New Guinea } & \\ \text { Russia } & \end{array}$

Natural gas

Turkmenistan

Aluminum

Tajikistan

Copper

Mongolia

Kazakhstan

Papua New Guinea

Gold

Papua New Guinea

Uzbekistan

Timber (Asian

Lao P.D.R.

Cambodia

hardwood)

Solomon Islands

Indonesia

Myanmar

Papua New Guinea

Timber (softwood)

Latvia

New Zealand

Cotton

Pakistan

Azerbaijan

Uzbekistan

Turkmenistan

Tajikistan

Jute

Bangladesh

Livestock

New Zealand

Fish

Maldives

Solomon Is.

Copra and
coconut oil

Source: International Monetary Fund.

Note: Trade data denominated in local currency was converted to U.S. dollars using the periodaverage exchange rate.

root test has low power. The low power problem means that a failure to reject the null hypothesis of a unit root cannot be construed as providing evidence for accepting the null. While there is little that can be done to improve the power of unit root tests, an interval estimate of the autoregressive parameter does yield useful information as to whether a failure to reject the null hypothesis of a unit root is due to the null being true or due to the uncertainty of the estimate of the autoregressive parameter.

Second, using unbiased estimates of the autoregressive parameter we calculate scalar measures of the duration (in terms of the number of periods) of typical price shocks, and the exact confidence interval surrounding the estimated median duration 
of shocks. Importantly, the confidence intervals are useful in their own right, as indicators of the variability of the persistence of shocks. The median-unbiased measures we use are potentially more informative to policymakers as to the persistence of shocks in comparison with either (i) analyses of the trend-stationary or differencestationary dichotomy of standard unit root tests, which focus only on whether shocks are mean-reverting (finite persistence) or not (infinite persistence); or (ii) nonparametric estimators, such as Cochrane's (1988) variance ratio, which provides information only on the share of the long-run variance in the total variance of price shocks. Third, we extend the results of Andrews (1993). Using Monte Carlo methods we estimate the median and 90 percent confidence interval of the median function of the autoregressive/unit root model, for those cases when there are between 300 and 500 observations. Fourth, unlike earlier studies, we focus our attention on the duration of shocks to individual commodities, rather than aggregate indices, as the persistence of shocks to aggregated series can differ greatly from the persistence of shocks to individual time series. ${ }^{2}$

We find that, on average, shocks to commodity prices are very long-lasting. For the majority of individual commodities it typically takes more than five years for half of the effect of the initial shock to dissipate. Moreover, the confidence intervals surrounding the estimated median duration of price shocks are typically quite wide, indicating that the persistence of shocks is variable in length. Unlike previous work, which claimed that finite persistence indicates that commodity stabilization schemes can be successful in stabilizing export revenues (León and Soto, 1997), our unbiased scalar measures of persistence indicate that there are many commodities for which shocks are finite, yet very long-lived, which casts doubt on the efficacy of stabilization schemes.

Our results highlight the need for policymakers to be cautious when implementing schemes designed to ameliorate the domestic effects of shocks to world commodity prices. In using policies such as national stabilization arrangements (through buffer stock schemes), international stabilization arrangements (through international commodity agreements), or compensatory financing, knowledge of the typical duration of price shocks is crucial. If price shocks are typically short-lived, then scope exists for policy initiatives to smooth national income and consumption. Alternatively, if price shocks are typically long-lived, then adjustment to the new long-run levels of national consumption and income is the preferred policy response. Our findings indicate that, for those commodities which typically experience highly persistent price shocks, caution should be exhibited in implementing these schemes, as for long-lasting shocks it is more likely that their associated storage, financing, and output-reduction costs will exceed the consumption- or income-smoothing benefits flowing from the smoothing of commodity prices.

The remainder of the paper is organized as follows. Section I sets out a median-unbiased estimator of first-order autoregressive/unit root (AR/UR)

\footnotetext{
${ }^{2}$ We also examine the extent to which the change in nominal exchange regime in the early 1970s has altered the persistence of shocks to commodity prices. When comparing how long it takes for a shock to dissipate, the findings of this paper are suggestive of greater persistence in the flexible exchange rate regime than in the fixed exchange rate regime (see Appendix II).
} 
models, and its advantages as a measure of the persistence of shocks to economic time series. Section II describes the data used in the study, while Section III presents the main empirical findings regarding the persistence of shocks to commodity prices during the period 1957 to 1998 . Several implications for public policy of the findings regarding the persistence of commodity price shocks are discussed in Section IV. Some concluding comments are contained in Section V.

\section{Measuring Persistence of Shocks}

The median-unbiased estimation procedure proposed by Andrews (1993), rather than unit root tests, is used to determine the persistence of shocks to commodity prices. Standard unit root testing procedures (such as those of Dickey and Fuller, 1979, and Phillips and Perron, 1988) suffer from two main disadvantages: (i) the least squares estimates of the autoregressive parameter in unit root regressions will be biased toward zero (Orcutt, 1948); and (ii) they have low power against plausible trend-stationary alternatives (DeJong and others, 1992). The downward bias in least squares estimates of the autoregressive parameter arises because there is an asymmetry in the distribution of estimators of the autoregressive parameter in AR/UR models (the distribution is skewed to the left, resulting in the median exceeding the mean). As a result, the median is a better measure of central tendency than the mean in least squares estimates of AR/UR models. In addition, if standard unit root tests fail to reject the null, then, because of low power problems, nothing can be confidently implied about the persistence of shocks to the series.

The median-unbiased estimator of Andrews combines unbiasedness with the use of point and interval estimators in achieving a more accurate estimate of the persistence of shocks to economic time series. Interval estimation addresses the power problem by informing us whether we are failing to reject the null because it is true or because there is too much uncertainty. The bias correction delivers an impartiality property to the decision-making process, because there is an equal chance of under- or overestimating the autoregressive parameter in the unit root regression, and thus the probability of selecting the correct model is at least as large as the probability of selecting the incorrect model. Moreover, an unbiased estimate of the autoregressive coefficient will allow us to calculate an unbiased estimate of the persistence of shocks. ${ }^{3}$

The Andrews (1993) median-unbiased estimator is concerned with the estimation of first-order AR/UR models with independent identically distributed normal errors. The model of the time series $\left\{Y_{t}: t=0, \ldots, T\right\}$ considered is that which includes an intercept and time trend: 4

\footnotetext{
${ }^{3}$ The extension of these median-unbiased procedures to $p$ th-order processes is considered in Andrews and Chen (1994); for AR( $p)$ models the procedures are no longer exact, only approximate. Unbiased unit root tests have been previously developed by Stock (1991) and Rudebusch (1992), both for $p$ th-order autoregressive processes with a time trend. See Andrews and Chen (1994) for a description of how these tests compare and contrast with the median-unbiased procedures.

${ }^{4}$ In equation (1), $\varepsilon_{t} \sim$ iid $N\left(0, \sigma^{2}\right)$ for $\sigma^{2}>0, Y_{0} \sim N\left(\mu, \sigma^{2} /\left(1-\alpha^{2}\right)\right)$ if $\alpha \in(-1,1)$, and $Y_{0}$ is an arbitrary constant or random variable if $\alpha=1$.
} 


$$
\begin{aligned}
& Y_{t}=\mu^{*}+\alpha Y_{t-1}+\beta^{*} t+\varepsilon_{t} \text { for } t=1, \ldots, T, \\
& \text { where } \mu^{*}=\mu(1-\alpha)+\alpha \beta, \beta^{*}=\beta(1-\alpha), \text { and } \alpha \in(-1,1]
\end{aligned}
$$

where $\varepsilon_{t}$ are the innovations of the model, $\mu$ is the intercept and $t$ is the trend.

To calculate the median-unbiased estimator, suppose $\hat{\alpha}$ is an estimator (of the autoregressive parameter, $\alpha$ ) whose median function $(m(\alpha))$ is uniquely defined $\forall \alpha \in(-1,1]$, then $\hat{\alpha}_{u}$ (the median-unbiased estimator of $\alpha$ ) is defined as:

$$
\hat{a}_{u}=\left\{\begin{array}{c}
1 \text { if } \hat{\alpha}>m(1) \\
m^{-1}(\hat{\alpha}) \text { if } m(-1)<\hat{\alpha} \leq m(1) \\
-1 \text { if } \hat{\alpha} \leq m(-1)
\end{array}\right.
$$

where $m(-1)=\lim _{\alpha \rightarrow-1} m(\alpha)$, and $m^{-1}:(m(-1), m(1)] \rightarrow(-1,1]$ is the inverse function of $m($.$) that satisfies m^{-1}(m(\alpha))=\alpha$ for $\alpha \in(-1,1]$. That is, if we have a function that for each true value of $\alpha$ yields the median value of $\hat{\alpha}$, then we can simply use the inverse function to obtain a median-unbiased estimate of $\alpha$. For example, if the least squares estimate of $\alpha$ equals 0.8 then we do not use that estimate, but instead use that value of $\alpha$ that results in the least squares estimator having a median of $0.8 .^{5,6}$ In addition, using the 0.05 and 0.95 quantile functions of $\hat{\alpha}$ we can construct two-sided 90 percent confidence intervals or one-sided 95 percent confidence intervals for $\alpha$. These confidence intervals can be used either to provide a measure of the accuracy of $\hat{\alpha}$ or to construct exact one- or two-sided tests of the null hypothesis that $\alpha=\alpha_{0}$. In this paper we use confidence intervals only to provide a measure of the accuracy of $\hat{\alpha} .^{7}$

The median, 0.05 quantile (lower band) and 0.95 quantile (upper band) of the function $m(\alpha)$ for the AR/UR model without and with constants and time trends has been computed for various sample sizes up to $T+1=200$ in Andrews (1993, Tables 1-3). In this paper we extend these results, using Monte Carlo simulations (of 10,000 replications) to estimate the median, lower band and upper band of the function of the AR coefficient for the model in equation (1), for sample sizes of $T+1=300, T+1=400$ and $T+1=500$ (see Appendix III). ${ }^{8}$ For sample sizes

${ }^{5}$ If the distribution of the least squares estimator of $\alpha$ depends only on $\alpha$ and is monotone in $\alpha$ (as in the $\mathrm{AR}(1)$ case), then the resultant estimator will have the property of median unbiasedness (Andrews, 1993).

${ }^{6}$ The size of the bias correction can be large, especially when $\alpha$ is close to one. For example, for a sample size of 60 observations and using the model of equation (1), a least squares estimate of $\alpha=0.85$ or greater would correspond to a median-unbiased estimate of $\alpha=1.00$; that is, $m(1)=0.85$.

${ }^{7}$ When conducting the unit root test (null hypothesis that $\alpha=1$ ) we prefer to use an unbiased model selection rule rather than the confidence interval. Such a rule recommends choosing a unit root model when the median-unbiased estimate of $\alpha$ equals 1 and choosing a trend stationary model if the medianunbiased estimate of $\alpha$ is less than 1.

${ }^{8}$ As noted by Andrews (1993, p. 163), for sample sizes greater than $T+1=200$, simulation methods (as used in this paper) will be superior to the numerical procedures used by Andrews to calculate the quantiles of the least squares estimators of $\hat{\alpha}$. 
neither given in the tables of Andrews (1993) nor calculated in this paper, $m(\alpha)$ can be obtained by interpolation.

To illustrate the use of the tables, consider the least squares estimator for the model in equation ( 1 ) and sample size $T+1=300$. Suppose the least squares estimate of the autoregressive parameter is 0.785 then, as shown in Appendix III, the median estimate is 0.80 , the 0.05 quantile is $0.86(0.785$ is 24 percent of the way between 0.772 and 0.827 , therefore we choose 24 percent of the way between 0.85 and 0.90 , which is 0.862 ), and the 0.95 quantile is 0.74 (0.785 is 39 percent of the way between 0.751 and 0.839 , therefore we choose 39 percent of the way between 0.70 and 0.80 , which is 0.739 ). Thus the two-sided 90 percent confidence interval is $(0.74,0.86)$.

Our interest in this paper concerns the persistence of shocks to economic time series. The measures we will use to quantify this persistence are the impulse response function (IRF), the cumulative impulse response function (CIR), and the half-life of a unit shock (HLS). The IRF of a time series $\left\{Y_{t}: t=1,2, \ldots\right\}$ measures the effect of a unit shock occurring at time $t$ (that is, $\varepsilon_{t} \rightarrow \varepsilon_{t}+1$ in equation (1)) on the values of $Y_{t}$ at the future time periods $t+1, t+2, \ldots$ The IRF quantifies the persistence of shocks to individual time series. For a series with a unit root, the IRF never dies out; however, for a trend-stationary series the IRF does die out. In any event, whether an individual time series is trend stationary or has a unit root, the relative magnitude of the IRF across different time horizons indicates the extent of the persistence of shocks to the individual series. The IRF is defined as:

$$
\operatorname{IRF}(\tau)=\alpha^{\tau} \text { for } \tau=0,1,2, \ldots
$$

Rather than consider the whole IRF, Andrews (1993) provides two scalar measures of persistence that summarize the impulse response function: the cumulative impulse response (CIR) and the half-life of a unit shock (HLS). Whereas the CIR gives the total cumulative effect of a unit shock on the entire future of a time series (that is, the sum of the IRF over all time horizons), for $\alpha \geq 0$ the HLS gives the length of time until the impulse response of a unit shock is half its original magnitude. These measures are defined as:

$$
C I R=\sum_{\tau=0}^{\infty} \operatorname{IRF}(\tau)=(1-\alpha)^{-1} \text { and } H L S=A B S(\log (1 / 2) / \log (\alpha)) .
$$

Exactly median-unbiased estimators and exact confidence intervals are also calculated for the IRF, CIR, and HLS, which provide information on the variability of the scalar measures of persistence. The median-unbiased estimate for these measures of persistence is calculated by using the median-unbiased estimate of $\alpha$ in the formulae of equation (4). Similarly, the two-sided 90 percent confidence interval is calculated using the 0.05 and 0.95 quantiles in the formulae of equation (4). ${ }^{9}$

\footnotetext{
${ }^{9}$ These median-unbiased measures can be compared with least squares estimators of persistence and associated confidence intervals, where the least squares estimates will (given they are functions of a biased $\alpha$ ) tend to understate the actual amount of persistence in shocks to economic time series (see Section III and Table 2 below).
} 


\section{Data}

In this study of the persistence of shocks to commodity prices, we use monthly commodity price data taken from the nominal price indices reported in the International Monetary Fund's International Financial Statistics (IFS) for the period January 1957 to December 1998. The data used in this study are real commodity price series, formed as the ratio of the chosen IFS nominal commodity index (defined in U.S. dollars per unit) deflated by the index of manufacturing unit values (base year 1990). ${ }^{10}$ The nominal commodity indices are for 44 individual commodities, 8 subaggregates of similar commodity indices (cereals, vegetable oils and protein meals, meat, coffee, timber, hardwoods, softwoods, and wool), and 8 aggregations of particular indices (all commodities, nonfuel commodities, agricultural raw materials, beverages, fertilizer, foods, metals, and sugar). ${ }^{11}$ The data are expressed in logarithmic form.

The definition and derivation of each nominal commodity index is given in Appendix I. As to descriptive statistics of the real commodity-price data, for most of the price series there is evidence of significant skewness. All commodities display significant leptokurtosis, with tails of their distribution much thicker than those of the normal distribution (Cashin, McDermott, and Scott, 1999). These results are consistent with the earlier findings of Deaton and Laroque (1992).

The all commodities index and its components (nonfuel commodities and petroleum) are presented in Figure 1. This shows the rapid rise in petroleum prices in the 1970s and their downward movement in the mid-1980s, the gradual fall in commodity prices since the peaks of the early 1970s and mid-1980s, and the decline to record lows at end-1998; it also illustrates the increasing volatility of commodity prices since the early 1970s. Figures 2 and 3 depict the recent evolution of petroleum prices (which have returned to their pre-oil-shock levels) and nonfuel commodities (which have fallen to near record lows since their recent peak in early 1997).

Each of the eight aggregated price indices is presented in Figure 4, as well as the petroleum price index, in both real and nominal (U.S. dollar) terms. Noticeable in many figures is the jump in nominal prices in 1972, following the depreciation of the dollar. There appears to be a tendency for the price indices to trend downward over the period, particularly for nonfuel commodities, beverages, metals, and food. Many of the price indices also exhibit a good deal of year-to-year variability. For example, the evolution of the beverages index is highlighted by the supply shock of the mid-1970s (owing to a severe Brazilian frost in 1975 that sharply cut available stocks). Sharp price movements in other indices are often associated with the formation and suspension of international commodity agreements designed to support world commodity prices. The petroleum index displays the spikes in

\footnotetext{
${ }^{10}$ The manufacturing unit value (MUV) index is a unit value index of exports from 20 industrial countries. Use of the MUV index as a deflator is common to most studies in the commodity price literature (see Appendix I for details).

${ }^{11}$ The aggregate indices are constructed by weighting indices for the individual commodity price series by the average export earnings for the commodities selected, using the 1987-89 average world export earnings to derive the weights. The weights and further details are provided in Appendix I.
} 
Figure 1. Prices of Primary Commodities, 1957:1-1998:12

(log of real price indices, $1990=100$ )

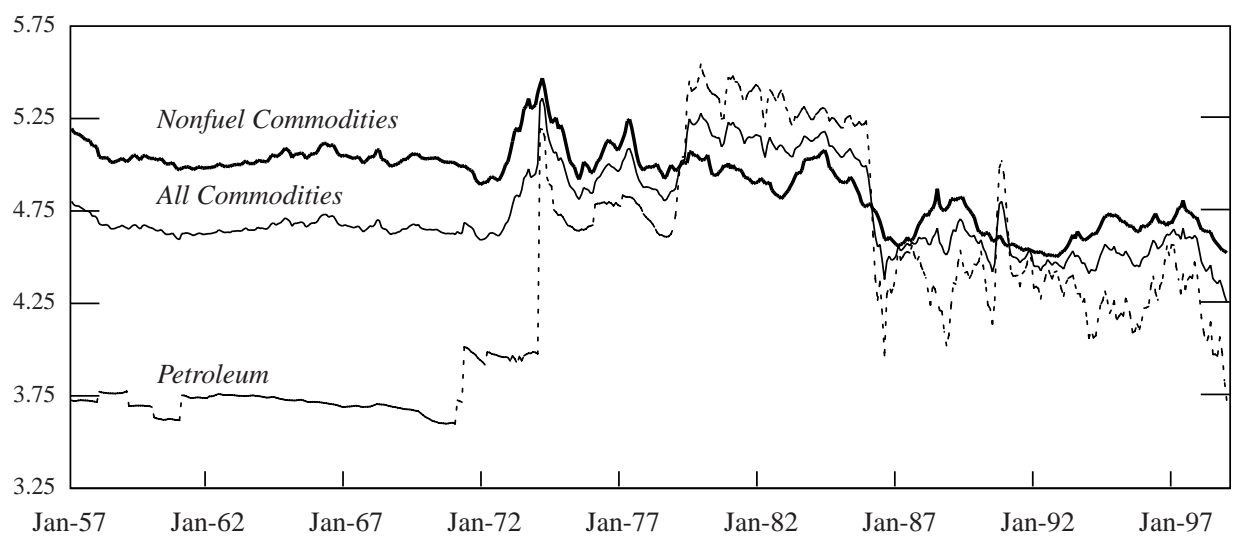

Figure 2. Spot Prices of Crude Petroleum, 1997-98

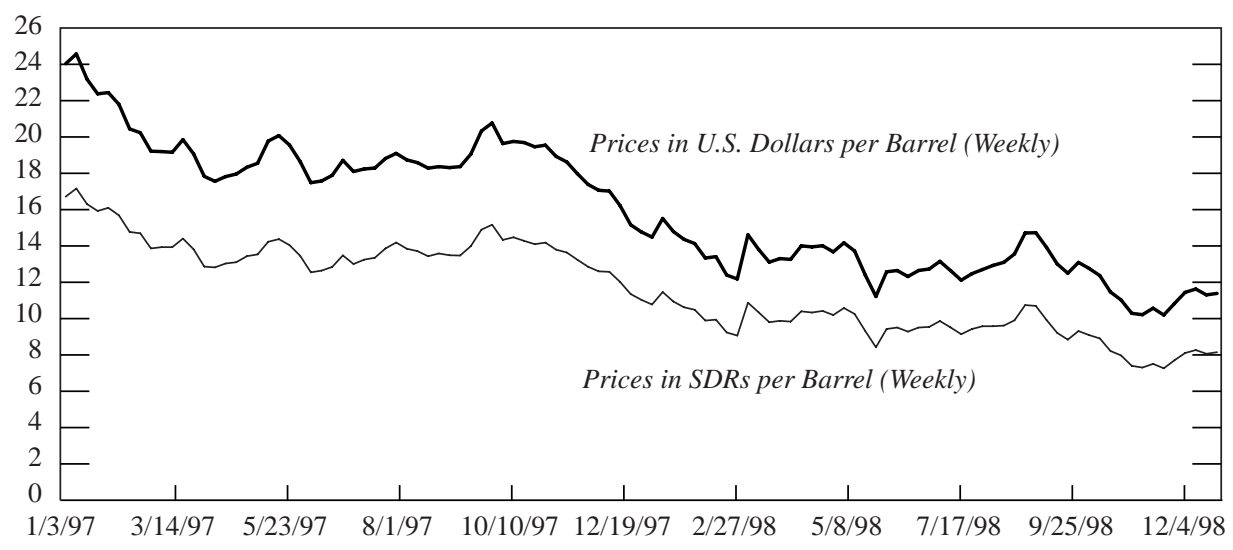

Figure 3. Index of Prices of Nonfuel Commodities, 1997-98

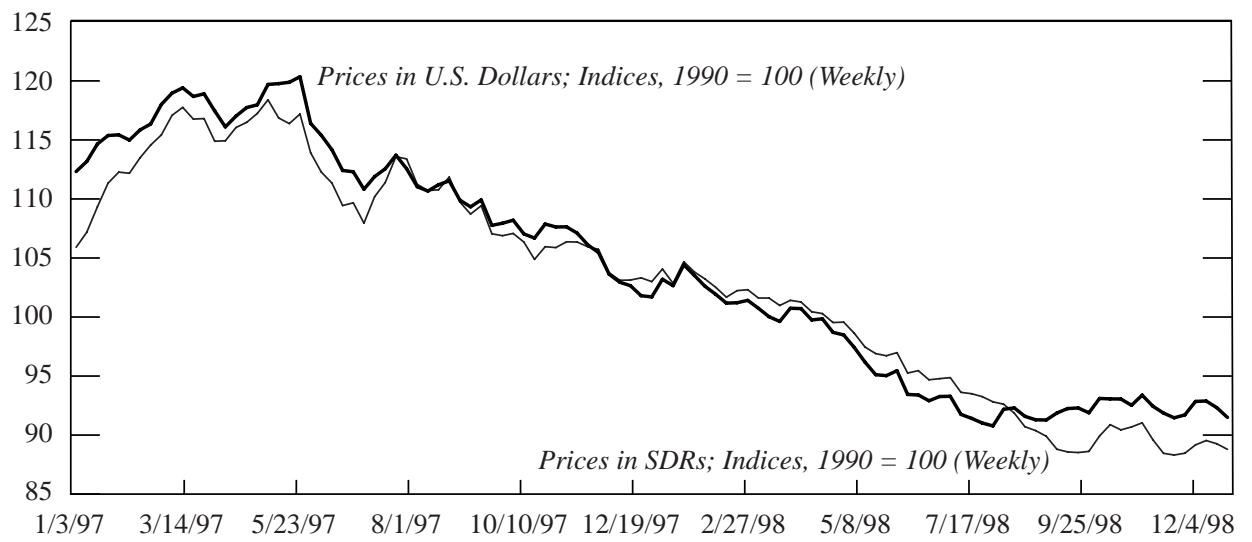


Figure 4. Prices of Primary Commodities

All Commodities Price Index, 1957:1 - 1998:12

$($ nominal and real terms, $1990=100)$

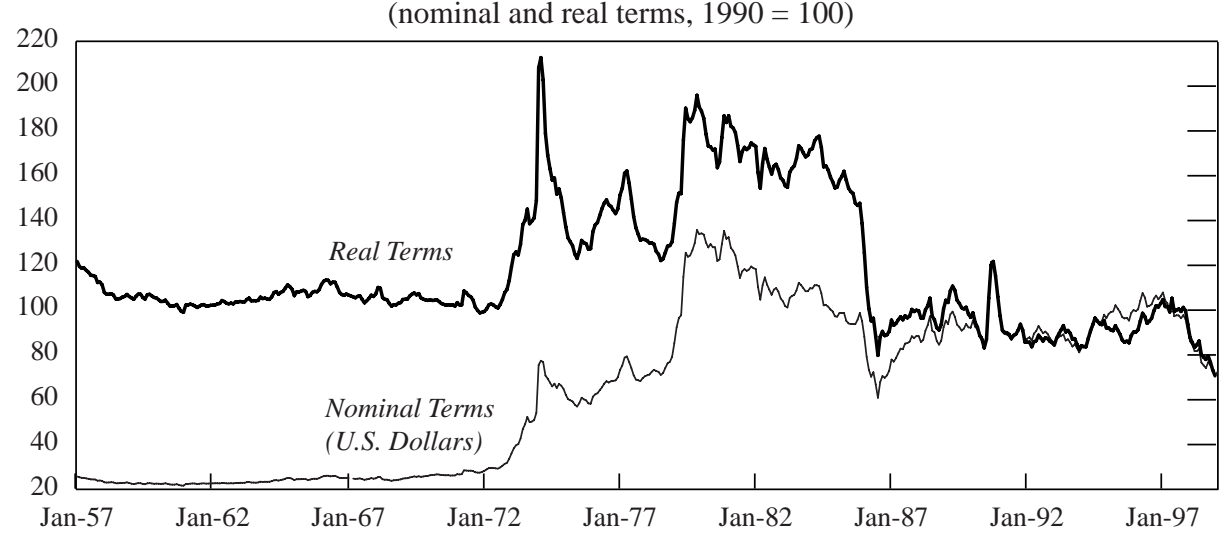

Nonfuel Commodities Price Index, 1957:1 - 1998:12

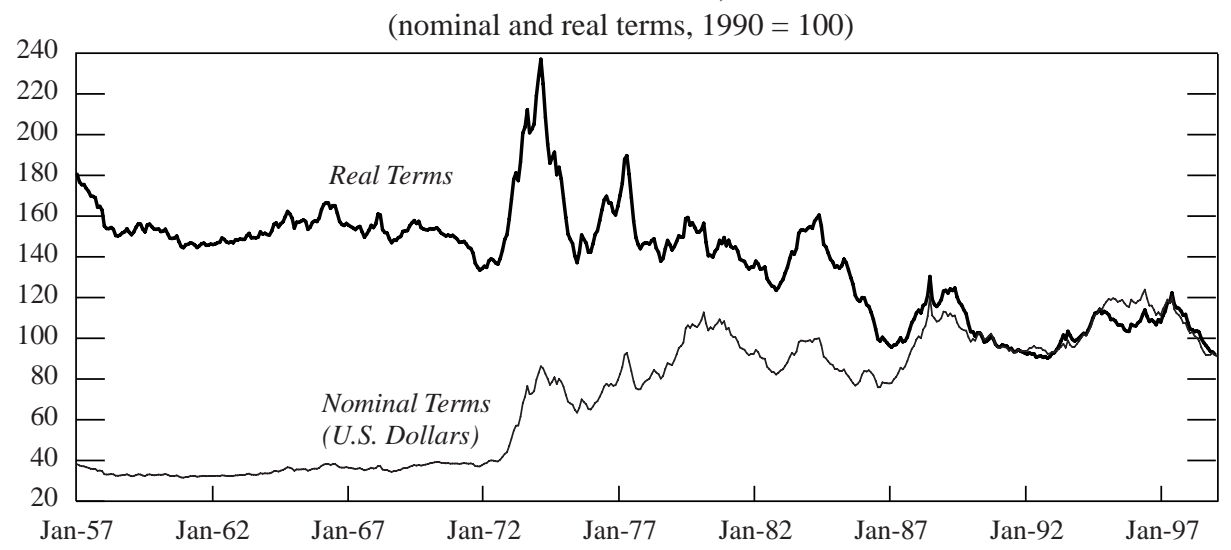

Beverages Price Index, 1957:1 - 1998:12

$($ nominal and real terms, $1990=100)$

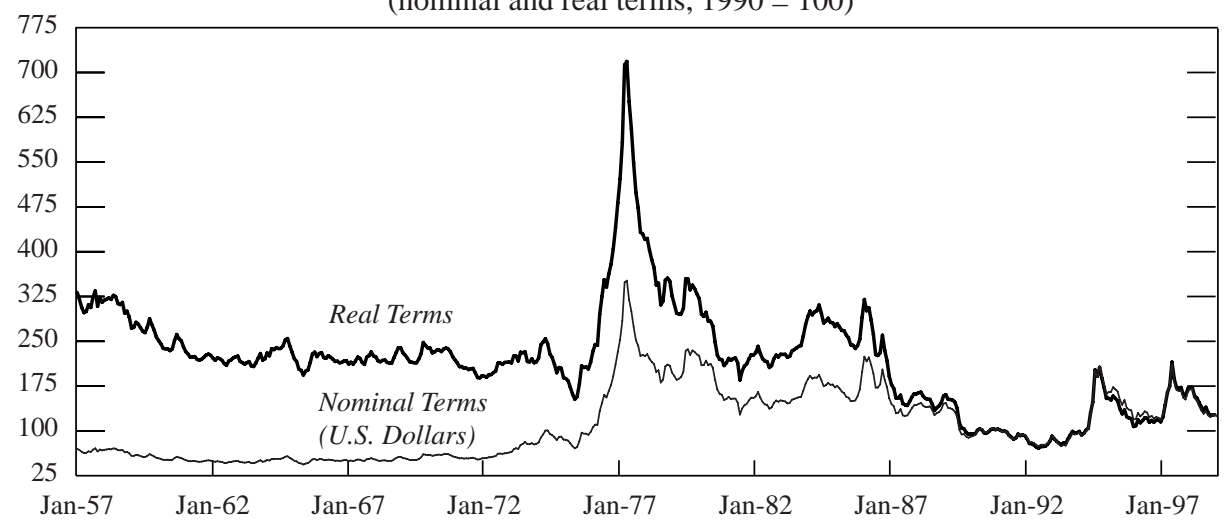


Figure 4. (continued)

Raw Materials Price Index, 1957:1 - 1998:12

(nominal and real terms, $1990=100)$

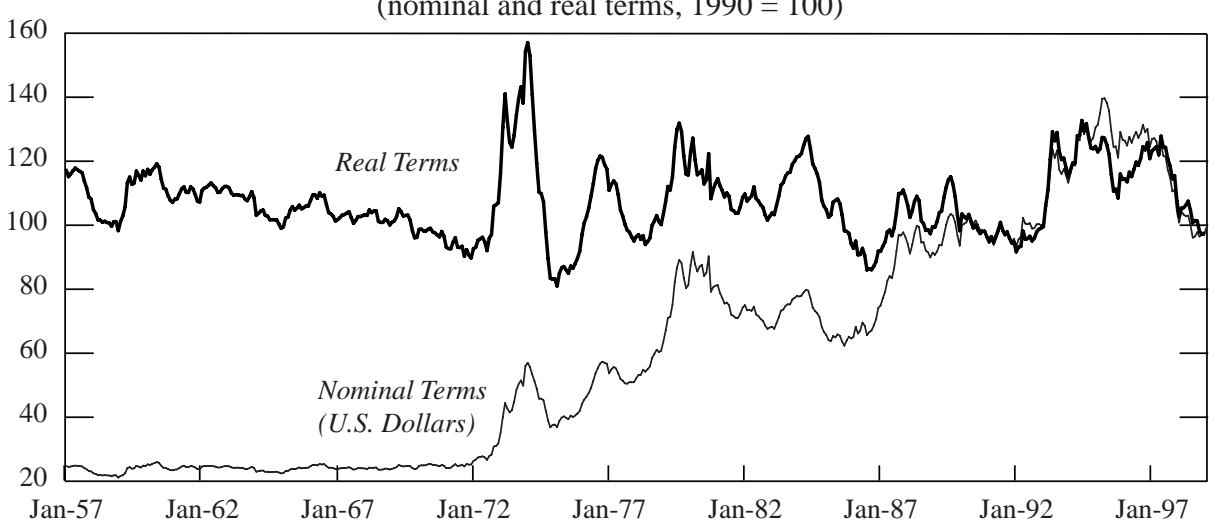

Metals Price Index, 1957:1 - 1998:12

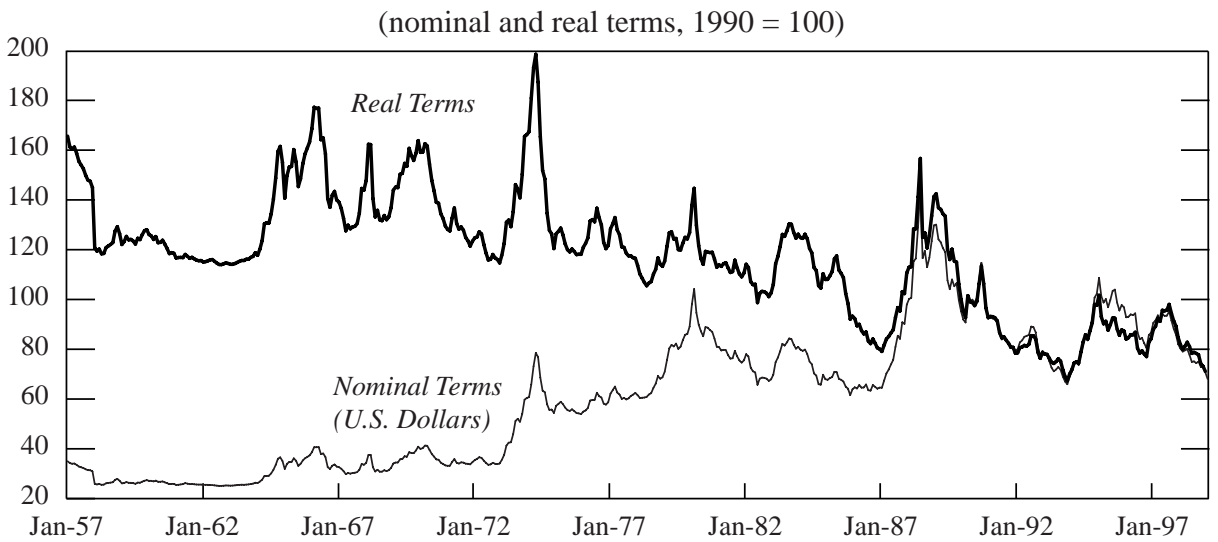

Food Price Index, 1957:1 - 1998:12

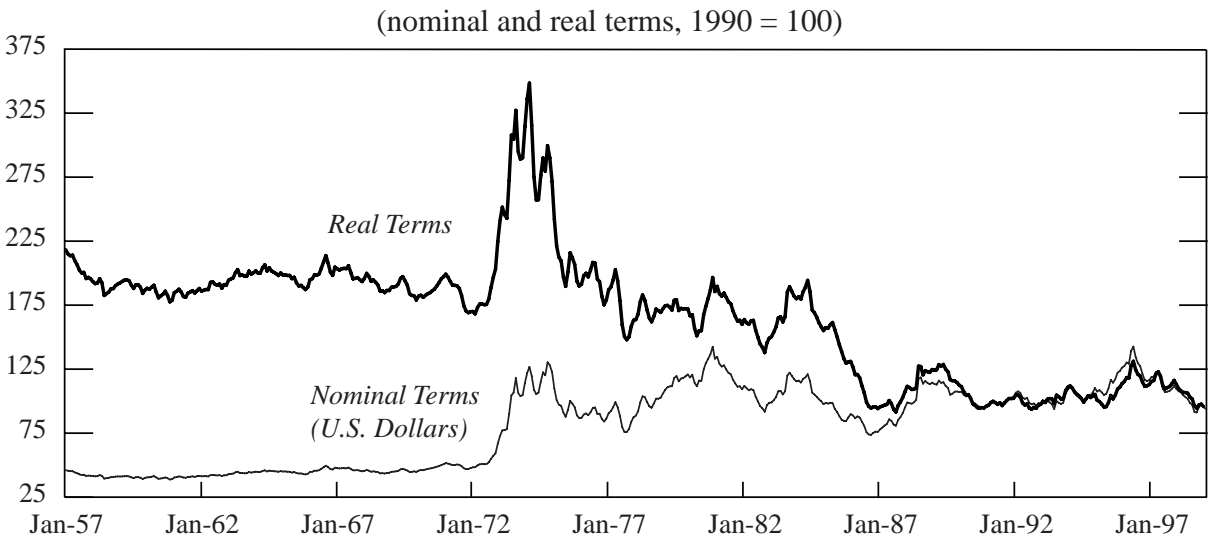


Figure 4. (concluded)

Fertilizer Price Index, 1957:1 - 1998:12

(nominal and real terms, $1990=100)$

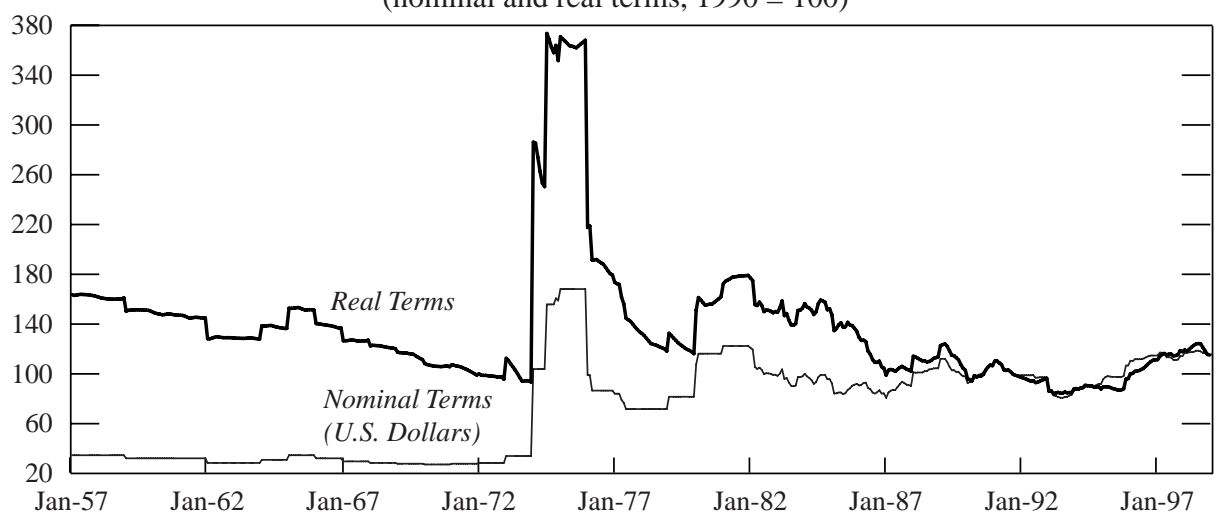

Petroleum Price Index, 1957:1 - 1998:12

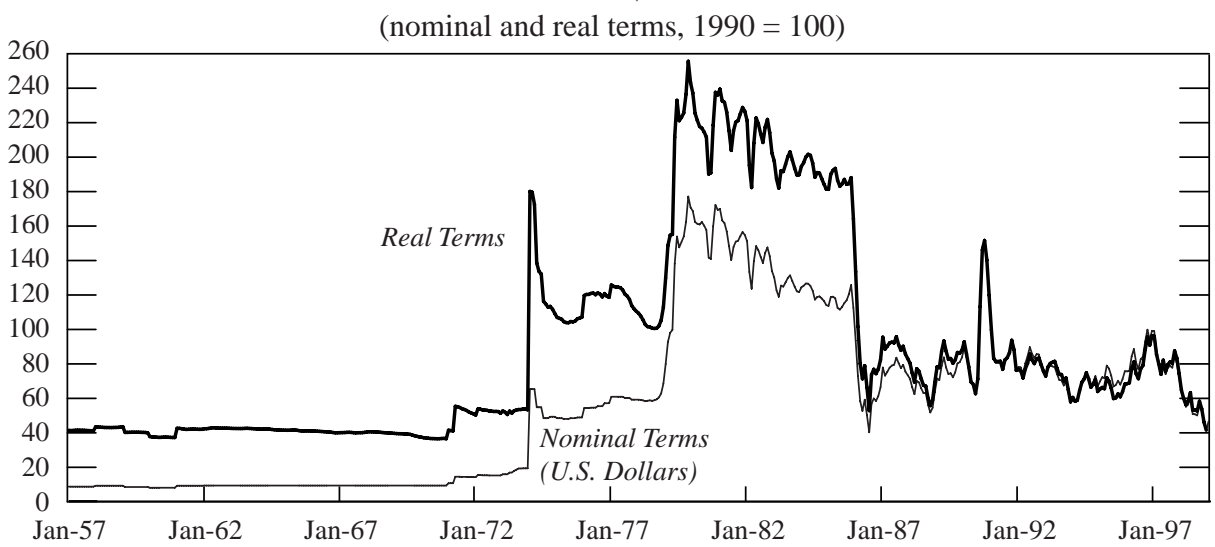

Sugar Price Index, 1957:1 - 1998:12

(nominal and real terms, $1990=100)$

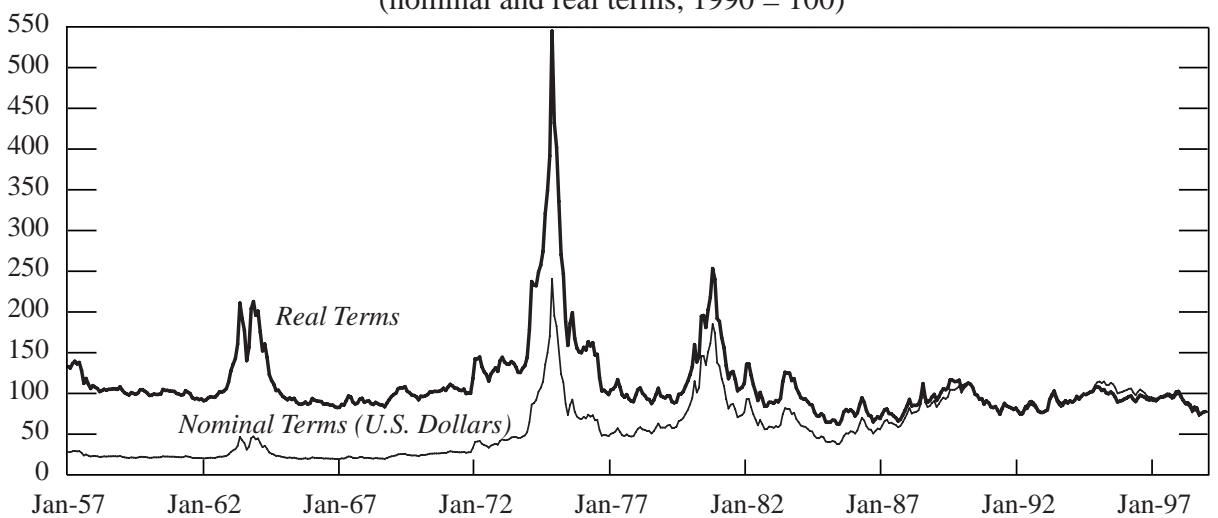


prices associated with the Organization for Petroleum Exporting Countries (OPEC) oil shocks of 1973 and 1979, while the fall in the metals index after 1985 is associated with a general lack of demand, expanding production, and the collapse of the International Tin Agreement. The peaks in the sugar index in 1963, 1974, and 1980 reflect, respectively, the suspension of the International Sugar Agreement, a rapid decline in the ratio of world stocks to sugar consumption, and adverse supply shocks in key exporting countries. The peaks in the food, raw materials, and metals indices in 1973-74 partly reflect the influence of adverse supply shocks, but are also largely attributable to strong demand for commodities from fast-growing industrialized countries, prior to the oil-shock-induced recessions of the 1970s (IMF, 1986).

\section{Empirical Results}

In this Section we apply the median-unbiased procedures to the series of world real commodity prices described in Section II. The 60 commodity price series are analyzed using Andrews' (1993) model (equation (1)). Table 2 sets out the results. As an example of how to interpret the table, we take the particular cases of tea, coarse wool, and tobacco. For tea, the median-unbiased (MU) estimate of $\alpha$ is 0.936 , as opposed to the least squares (LS) estimate of 0.926 . While the difference between these two estimates is small in absolute terms, this has a relatively large effect on calculations of the persistence of the shocks. The length of the 90 percent confidence interval for the median-unbiased estimate of $\alpha(0.906,0.967)$ indicates that $\alpha$ is estimated fairly precisely. The IRF declines monotonically toward zero (that is, the IRF eventually dies out). The estimates yield a [LS 13.514; MU 15.625] 16 percent increase in the estimate of CIR (the total cumulative effect of a unit shock on the future evolution of the price series). This indicates that a unit shock to tea generates an eventual $(t=\infty) 15.625$ unit displacement of the level of the price of tea. Moreover, the time it takes for half of the impulse response of a shock to the price of tea to dissipate is 9.016 months for the LS estimate and 10.480 months for the MU estimate. Accordingly, shocks to tea do not appear to be particularly persistent.

Alternatively, for coarse wool the median-unbiased estimate of $\alpha$ is 0.990 , as opposed to the least squares estimate of 0.976 . The IRF declines monotonically toward zero, yet does not die out. The length of the 90 percent confidence interval for the MU estimate of $\alpha(0.969,1.00)$ indicates that $\alpha$ is estimated fairly precisely (and with more precision than for tea). Moreover, the LS estimate of $\alpha$ is relatively close to the lower bound of the MU estimate's 90 percent confidence interval, which indicates that the series for coarse wool exhibits large differences between the LS and median-unbiased measures of persistence to shocks. The estimates yield a [LS 41.667; MU 100.724] 142 percent increase in the estimate of CIR (the total cumulative effect of a unit shock on the future evolution of the price series). This indicates that a unit shock to coarse wool generates an eventual $(t=\infty)$ 100.724 unit displacement of the level of the price of wool. Moreover, the time it takes for half of the impulse response of a shock to the price of coarse wool to dissipate rises from 28.533 months for the LS estimate to 69.649 months for the 
Table 2. Results of Median-Unbiased Autoregressive/ Unit Root Estimation, Commodity Price Indices, 1957:1-1998:12

\begin{tabular}{|c|c|c|c|c|c|c|c|}
\hline Commodity & Estimator & $\alpha$ & $\operatorname{IR}(4)$ & $\operatorname{IR}(12)$ & $\operatorname{IR}(36)$ & CIR & HLS \\
\hline All commodities* & $\begin{array}{l}\text { LS } \\
\text { Median- } \\
\text { Unbiased }\end{array}$ & $\begin{array}{c}0.993 \\
1.00 \\
{[.995-1.00]}\end{array}$ & $\begin{array}{c}0.972 \\
1.00 \\
{[.981-1.00]}\end{array}$ & $\begin{array}{c}0.919 \\
1.00 \\
{[.943-1.00]}\end{array}$ & $\begin{array}{c}0.777 \\
1.00 \\
{[.839-1.00]}\end{array}$ & $\begin{array}{c}142.857 \\
\infty \\
{[205.590-\infty]}\end{array}$ & $\begin{array}{c}98.674 \\
\infty \\
{[142.147-\infty]}\end{array}$ \\
\hline Nonfuel & $\begin{array}{l}\text { LS } \\
\text { Median- } \\
\text { Unbiased }\end{array}$ & $\begin{array}{c}0.986 \\
1.00 \\
{[.984-1.00]}\end{array}$ & $\begin{array}{c}0.945 \\
1.00 \\
{[.936-1.00]}\end{array}$ & $\begin{array}{c}0.844 \\
1.00 \\
{[.819-1.00]}\end{array}$ & $\begin{array}{c}0.602 \\
1.00 \\
{[.550-1.00]}\end{array}$ & $\begin{array}{c}71.429 \\
\infty \\
{[60.753-\infty]}\end{array}$ & $\begin{array}{c}49.163 \\
\infty \\
{[41.763-\infty]}\end{array}$ \\
\hline Food & $\begin{array}{l}\text { LS } \\
\text { Median- } \\
\text { Unbiased }\end{array}$ & $\begin{array}{c}0.985 \\
1.00 \\
{[.983-1.00]}\end{array}$ & $\begin{array}{c}0.941 \\
1.00 \\
{[.932-1.00]}\end{array}$ & $\begin{array}{c}0.834 \\
1.00 \\
{[.810-1.00]}\end{array}$ & $\begin{array}{c}0.580 \\
1.00 \\
{[.531-1.00]}\end{array}$ & $\begin{array}{c}66.667 \\
\infty \\
{[57.342-\infty]}\end{array}$ & $\begin{array}{c}45.862 \\
\infty \\
{[39.399-\infty]}\end{array}$ \\
\hline Cereals & $\begin{array}{l}\text { LS } \\
\text { Median- } \\
\text { Unbiased }\end{array}$ & $\begin{array}{c}0.983 \\
1.00 \\
{[.979-1.00]}\end{array}$ & $\begin{array}{c}0.934 \\
1.00 \\
{[.919-1.00]}\end{array}$ & $\begin{array}{c}0.814 \\
1.00 \\
{[.777-1.00]}\end{array}$ & $\begin{array}{c}0.539 \\
1.00 \\
{[.469-1.00]}\end{array}$ & $\begin{array}{c}58.823 \\
\infty \\
{[48.016-\infty]}\end{array}$ & $\begin{array}{c}40.426 \\
\infty \\
{[32.934-\infty]}\end{array}$ \\
\hline Maize & $\begin{array}{l}\text { LS } \\
\text { Median- } \\
\text { Unbiased }\end{array}$ & $\begin{array}{c}0.974 \\
0.987 \\
{[.966-1.00]}\end{array}$ & $\begin{array}{c}0.899 \\
0.951 \\
{[.870-1.00]}\end{array}$ & $\begin{array}{c}0.729 \\
0.859 \\
{[.659-1.00]}\end{array}$ & $\begin{array}{c}0.387 \\
0.634 \\
{[.287-1.00]}\end{array}$ & $\begin{array}{c}38.462 \\
79.518 \\
{[29.312-\infty]}\end{array}$ & $\begin{array}{c}26.311 \\
54.770 \\
{[19.969-\infty]}\end{array}$ \\
\hline Rice & $\begin{array}{l}\text { LS } \\
\text { Median- } \\
\text { Unbiased }\end{array}$ & $\begin{array}{c}0.978 \\
0.993 \\
{[.972-1.00]}\end{array}$ & $\begin{array}{c}0.915 \\
0.974 \\
{[.891-1.00]}\end{array}$ & $\begin{array}{c}0.767 \\
0.923 \\
{[.707-1.00]}\end{array}$ & $\begin{array}{c}0.449 \\
0.786 \\
{[.354-1.00]}\end{array}$ & $\begin{array}{c}45.455 \\
149.782 \\
{[35.125-\infty]}\end{array}$ & $\begin{array}{c}31.159 \\
103.481 \\
{[23.998-\infty]}\end{array}$ \\
\hline Wheat & $\begin{array}{l}\text { LS } \\
\text { Median- } \\
\text { Unbiased }\end{array}$ & $\begin{array}{c}0.971 \\
0.984 \\
{[0.963-1.00]}\end{array}$ & $\begin{array}{c}0.889 \\
0.939 \\
{[.859-1.00]}\end{array}$ & $\begin{array}{c}0.702 \\
0.828 \\
{[.633-1.00]}\end{array}$ & $\begin{array}{c}0.347 \\
0.568 \\
{[.253-1.00]}\end{array}$ & $\begin{array}{c}34.483 \\
64.182 \\
{[26.730-\infty]}\end{array}$ & $\begin{array}{c}23.553 \\
44.140 \\
{[18.179-\infty]}\end{array}$ \\
\hline $\begin{array}{l}\text { Vegetable oils } \\
\text { and protein } \\
\text { meals }\end{array}$ & $\begin{array}{l}\text { LS } \\
\text { Median- } \\
\text { Unbiased }\end{array}$ & $\begin{array}{c}0.975 \\
0.989 \\
{[.968-1.00]}\end{array}$ & $\begin{array}{c}0.904 \\
0.958 \\
{[.878-1.00]}\end{array}$ & $\begin{array}{c}0.738 \\
0.878 \\
{[.676-1.00]}\end{array}$ & $\begin{array}{c}0.402 \\
0.678 \\
{[.309-1.00]}\end{array}$ & $\begin{array}{l}40 \\
92.966 \\
{[31.133-\infty]}\end{array}$ & $\begin{array}{c}27.378 \\
64.092 \\
{[21.231-\infty]}\end{array}$ \\
\hline $\begin{array}{l}\text { Coconut } \\
\text { oil }\end{array}$ & $\begin{array}{l}\text { LS } \\
\text { Median- } \\
\text { Unbiased }\end{array}$ & $\begin{array}{c}0.976 \\
0.990 \\
{[.969-1.00]}\end{array}$ & $\begin{array}{c}0.907 \\
0.961 \\
{[.881-1.00]}\end{array}$ & $\begin{array}{c}0.747 \\
0.888 \\
{[.684-1.00]}\end{array}$ & $\begin{array}{c}0.417 \\
0.701 \\
{[.320-1.00]}\end{array}$ & $\begin{array}{c}41.667 \\
101.700 \\
{[32.092-\infty]}\end{array}$ & $\begin{array}{c}28.533 \\
70.146 \\
{[21.896-\infty]}\end{array}$ \\
\hline Fish meal & $\begin{array}{l}\text { LS } \\
\text { Median- } \\
\text { Unbiased }\end{array}$ & $\begin{array}{c}0.971 \\
0.985 \\
{[.963-1.00]}\end{array}$ & $\begin{array}{c}0.889 \\
0.940 \\
{[.859-1.00]}\end{array}$ & $\begin{array}{c}0.702 \\
0.830 \\
{[.634-1.00]}\end{array}$ & $\begin{array}{c}0.347 \\
0.572 \\
{[.255-1.00]}\end{array}$ & $\begin{array}{c}34.483 \\
64.874 \\
{[26.861-\infty]}\end{array}$ & $\begin{array}{c}23.553 \\
44.620 \\
{[18.270-\infty]}\end{array}$ \\
\hline $\begin{array}{l}\text { Groundnut } \\
\text { oil }\end{array}$ & $\begin{array}{l}\text { LS } \\
\text { Median- } \\
\text { Unbiased }\end{array}$ & $\begin{array}{c}0.974 \\
0.988 \\
{[.967-1.00]}\end{array}$ & $\begin{array}{c}0.899 \\
0.953 \\
{[.873-1.00]}\end{array}$ & $\begin{array}{c}0.729 \\
0.867 \\
{[.666-1.00]}\end{array}$ & $\begin{array}{c}0.387 \\
0.651 \\
{[.295-1.00]}\end{array}$ & $\begin{array}{c}38.462 \\
84.338 \\
{[30.006-\infty]}\end{array}$ & $\begin{array}{c}26.311 \\
58.111 \\
{[20.450-\infty]}\end{array}$ \\
\hline Palm oil & $\begin{array}{l}\text { LS } \\
\text { Median- } \\
\text { Unbiased }\end{array}$ & $\begin{array}{c}0.975 \\
0.989 \\
{[.968-1.00]}\end{array}$ & $\begin{array}{c}0.904 \\
0.958 \\
{[.878-1.00]}\end{array}$ & $\begin{array}{c}0.738 \\
0.879 \\
{[.676-1.00]}\end{array}$ & $\begin{array}{c}0.402 \\
0.679 \\
{[.309-1.00]}\end{array}$ & $\begin{array}{l}40 \\
93.477 \\
{[31.196-\infty]}\end{array}$ & $\begin{array}{c}27.378 \\
64.446 \\
{[21.275-\infty]}\end{array}$ \\
\hline Soybean & $\begin{array}{l}\text { LS } \\
\text { Median- } \\
\text { Unbiased }\end{array}$ & $\begin{array}{c}0.965 \\
0.977 \\
{[.955-1.00]}\end{array}$ & $\begin{array}{c}0.867 \\
0.913 \\
{[.831-1.00]}\end{array}$ & $\begin{array}{c}0.652 \\
0.760 \\
{[.575-1.00]}\end{array}$ & $\begin{array}{c}0.277 \\
0.439 \\
{[.190-1.00]}\end{array}$ & $\begin{array}{c}28.571 \\
44.282 \\
{[22.178-\infty]}\end{array}$ & $\begin{array}{c}19.456 \\
30.346 \\
{[15.023-\infty]}\end{array}$ \\
\hline $\begin{array}{c}\text { Soybean } \\
\text { meal }\end{array}$ & $\begin{array}{l}\text { LS } \\
\text { Median- } \\
\text { Unbiased }\end{array}$ & $\begin{array}{c}0.962 \\
0.974 \\
{[.951-1.00]}\end{array}$ & $\begin{array}{c}0.856 \\
0.900 \\
{[.818-1.00]}\end{array}$ & $\begin{array}{c}0.628 \\
0.728 \\
{[.547-1.00]}\end{array}$ & $\begin{array}{c}0.248 \\
0.386 \\
{[.164-1.00]}\end{array}$ & $\begin{array}{c}26.316 \\
38.289 \\
{[20.422-\infty]}\end{array}$ & $\begin{array}{c}17.892 \\
26.192 \\
{[13.806-\infty]}\end{array}$ \\
\hline $\begin{array}{l}\text { Soybean } \\
\text { oil }\end{array}$ & $\begin{array}{l}\text { LS } \\
\text { Median- } \\
\text { Unbiased }\end{array}$ & $\begin{array}{c}0.973 \\
0.986 \\
{[.965-1.00]}\end{array}$ & $\begin{array}{c}0.896 \\
0.947 \\
{[.866-1.00]}\end{array}$ & $\begin{array}{c}0.720 \\
0.848 \\
{[.650-1.00]}\end{array}$ & $\begin{array}{c}0.373 \\
0.611 \\
{[.275-1.00]}\end{array}$ & $\begin{array}{c}37.037 \\
73.504 \\
{[28.374-\infty]}\end{array}$ & $\begin{array}{c}25.324 \\
50.601 \\
{[19.318-\infty]}\end{array}$ \\
\hline Meat & $\begin{array}{l}\text { LS } \\
\text { Median- } \\
\text { Unbiased }\end{array}$ & $\begin{array}{c}0.976 \\
0.990 \\
{[.968-1.00]}\end{array}$ & $\begin{array}{c}0.907 \\
0.959 \\
{[.879-1.00]}\end{array}$ & $\begin{array}{c}0.747 \\
0.881 \\
{[.678-1.00]}\end{array}$ & $\begin{array}{c}0.417 \\
0.685 \\
{[.312-1.00]}\end{array}$ & $\begin{array}{c}41.667 \\
95.484 \\
{[31.438-\infty]}\end{array}$ & $\begin{array}{c}28.533 \\
65.837 \\
{[21.442-\infty]}\end{array}$ \\
\hline Beef & $\begin{array}{l}\text { LS } \\
\text { Median- } \\
\text { Unbiased }\end{array}$ & $\begin{array}{c}0.974 \\
0.988 \\
{[.966-1.00]}\end{array}$ & $\begin{array}{c}0.899 \\
0.952 \\
{[.872-1.00]}\end{array}$ & $\begin{array}{c}0.729 \\
0.863 \\
{[.663-1.00]}\end{array}$ & $\begin{array}{c}0.387 \\
0.644 \\
{[.291-1.00]}\end{array}$ & $\begin{array}{c}38.462 \\
82.203 \\
{[29.705-\infty]}\end{array}$ & $\begin{array}{c}26.311 \\
56.631 \\
{[20.241-\infty]}\end{array}$ \\
\hline
\end{tabular}


Table 2. (continued)

\begin{tabular}{|c|c|c|c|c|c|c|c|}
\hline Commodity & Estimator & $\alpha$ & $\operatorname{IR}(4)$ & $\operatorname{IR}(12)$ & $\operatorname{IR}(36)$ & CIR & HLS \\
\hline Lamb & $\begin{array}{l}\text { LS } \\
\text { Median- } \\
\text { Unbiased }\end{array}$ & $\begin{array}{c}0.943 \\
0.953 \\
{[.926-.982]}\end{array}$ & $\begin{array}{c}0.791 \\
0.825 \\
{[.735-.931]}\end{array}$ & $\begin{array}{c}0.494 \\
0.562 \\
{[.397-.808]}\end{array}$ & $\begin{array}{c}0.121 \\
0.178 \\
{[.063-.527]}\end{array}$ & $\begin{array}{c}17.544 \\
21.353 \\
{[13.496-56.667]}\end{array}$ & $\begin{array}{c}11.811 \\
14.451 \\
{[9.004-38.931]}\end{array}$ \\
\hline Sugar & $\begin{array}{l}\text { LS } \\
\text { Median- } \\
\text { Unbiased }\end{array}$ & $\begin{array}{c}0.971 \\
0.985 \\
{[.963-1.00]}\end{array}$ & $\begin{array}{c}0.889 \\
0.939 \\
{[.859-1.00]}\end{array}$ & $\begin{array}{c}0.702 \\
0.829 \\
{[.634-1.00]}\end{array}$ & $\begin{array}{c}0.347 \\
0.570 \\
{[.254-1.00]}\end{array}$ & $\begin{array}{c}34.483 \\
64.573 \\
{[26.804-\infty]}\end{array}$ & $\begin{array}{c}23.553 \\
44.411 \\
{[18.230-\infty]}\end{array}$ \\
\hline $\begin{array}{c}\text { Sugar (free } \\
\text { market) }\end{array}$ & $\begin{array}{l}\text { LS } \\
\text { Median- } \\
\text { Unbiased }\end{array}$ & $\begin{array}{c}0.978 \\
0.994 \\
{[.972-1.00]}\end{array}$ & $\begin{array}{c}0.915 \\
0.976 \\
{[.893-1.00]}\end{array}$ & $\begin{array}{c}0.766 \\
0.931 \\
{[.713-1.00]}\end{array}$ & $\begin{array}{c}0.449 \\
0.806 \\
{[.362-1.00]}\end{array}$ & $\begin{array}{c}45.455 \\
167.578 \\
{[35.950-\infty]}\end{array}$ & $\begin{array}{c}31.159 \\
115.809 \\
{[24.571-\infty]}\end{array}$ \\
\hline Sugar (E.U.) & $\begin{array}{l}\text { LS } \\
\text { Median- } \\
\text { Unbiased }\end{array}$ & $\begin{array}{c}0.891 \\
0.901 \\
{[.865-.936]}\end{array}$ & $\begin{array}{c}0.630 \\
0.658 \\
{[.561-.707]}\end{array}$ & $\begin{array}{c}0.250 \\
0.285 \\
{[.117-.451]}\end{array}$ & $\begin{array}{c}0.016 \\
0.023 \\
{[.006-.092]}\end{array}$ & $\begin{array}{c}9.174 \\
10.062 \\
{[7.433-15.595]}\end{array}$ & $\begin{array}{c}6.006 \\
6.622 \\
{[4.797-10.460]}\end{array}$ \\
\hline Sugar (U.S.) & $\begin{array}{l}\text { LS } \\
\text { Median- } \\
\text { Unbiased }\end{array}$ & $\begin{array}{c}0.963 \\
0.975 \\
{[.952-1.00]}\end{array}$ & $\begin{array}{c}0.860 \\
0.903 \\
{[.821-1.00]}\end{array}$ & $\begin{array}{c}0.636 \\
0.735 \\
{[.554-1.00]}\end{array}$ & $\begin{array}{c}0.257 \\
0.398 \\
{[.170-1.00]}\end{array}$ & $\begin{array}{c}27.027 \\
39.549 \\
{[20.810-\infty]}\end{array}$ & $\begin{array}{c}18.385 \\
27.065 \\
{[14.075-\infty]}\end{array}$ \\
\hline Bananas & $\begin{array}{l}\text { LS } \\
\text { Median- } \\
\text { Unbiased }\end{array}$ & $\begin{array}{c}0.740 \\
0.748 \\
{[.698-.799]}\end{array}$ & $\begin{array}{c}0.299 \\
0.314 \\
{[.238-.408]}\end{array}$ & $\begin{array}{c}0.027 \\
0.031 \\
{[.013-.068]}\end{array}$ & $\begin{array}{c}0.00002 \\
0.000 \\
{[.000-.000]}\end{array}$ & $\begin{array}{c}3.846 \\
3.975 \\
{[3.314-4.986]}\end{array}$ & $\begin{array}{c}2.302 \\
2.392 \\
{[1.930-3.097]}\end{array}$ \\
\hline Beverages & $\begin{array}{l}\text { LS } \\
\text { Median- } \\
\text { Unbiased }\end{array}$ & $\begin{array}{c}0.988 \\
1.00 \\
{[.986-1.00]}\end{array}$ & $\begin{array}{c}0.952 \\
1.00 \\
{[.946-1.00]}\end{array}$ & $\begin{array}{c}0.865 \\
1.00 \\
{[.847-1.00]}\end{array}$ & $\begin{array}{c}0.648 \\
1.00 \\
{[.608-1.00]}\end{array}$ & $\begin{array}{c}83.333 \\
\infty \\
{[72.948-\infty]}\end{array}$ & $\begin{array}{c}57.415 \\
\infty \\
{[50.216-\infty]}\end{array}$ \\
\hline Coffee & $\begin{array}{l}\text { LS } \\
\text { Median- } \\
\text { Unbiased }\end{array}$ & $\begin{array}{c}0.984 \\
1.00 \\
{[.981-1.00]}\end{array}$ & $\begin{array}{c}0.938 \\
1.00 \\
{[.924-1.00]}\end{array}$ & $\begin{array}{c}0.824 \\
1.00 \\
{[.790-1.00]}\end{array}$ & $\begin{array}{c}0.560 \\
1.00 \\
{[.493-1.00]}\end{array}$ & $\begin{array}{c}62.500 \\
\infty \\
{[51.430-\infty]}\end{array}$ & $\begin{array}{c}42.974 \\
\infty \\
{[35.301-\infty]}\end{array}$ \\
\hline $\begin{array}{l}\text { Coffee } \\
\text { (other } \\
\text { milds) }\end{array}$ & $\begin{array}{l}\text { LS } \\
\text { Median- } \\
\text { Unbiased }\end{array}$ & $\begin{array}{c}0.979 \\
0.995 \\
{[0.973-1.00]}\end{array}$ & $\begin{array}{c}0.919 \\
0.982 \\
{[.898-1.00]}\end{array}$ & $\begin{array}{c}0.775 \\
0.946 \\
{[.724-1.00]}\end{array}$ & $\begin{array}{c}0.466 \\
0.847 \\
{[.379-1.00]}\end{array}$ & $\begin{array}{c}47.619 \\
216.950 \\
{[37.647-\infty]}\end{array}$ & $\begin{array}{c}32.659 \\
150.032 \\
{[25.747-\infty]}\end{array}$ \\
\hline $\begin{array}{l}\text { Coffee } \\
\text { (robusta) }\end{array}$ & $\begin{array}{l}\text { LS } \\
\text { Median- } \\
\text { Unbiased }\end{array}$ & $\begin{array}{c}0.988 \\
1.00 \\
{[.986-1.00]}\end{array}$ & $\begin{array}{c}0.953 \\
1.00 \\
{[.946-1.00]}\end{array}$ & $\begin{array}{c}0.865 \\
1.00 \\
{[.874-1.00]}\end{array}$ & $\begin{array}{c}0.648 \\
1.00 \\
{[.608-1.00]}\end{array}$ & $\begin{array}{c}83.333 \\
\infty \\
{[72.809-\infty]}\end{array}$ & $\begin{array}{c}57.415 \\
\infty \\
{[50.120-\infty]}\end{array}$ \\
\hline Cocoa beans & $\begin{array}{l}\text { LS } \\
\text { Median- } \\
\text { Unbiased }\end{array}$ & $\begin{array}{c}0.987 \\
1.00 \\
{[.986-1.00]}\end{array}$ & $\begin{array}{c}0.949 \\
1.00 \\
{[.943-1.00]}\end{array}$ & $\begin{array}{c}0.855 \\
1.00 \\
{[.839-1.00]}\end{array}$ & $\begin{array}{c}0.624 \\
1.00 \\
{[.591-1.00]}\end{array}$ & $\begin{array}{c}76.923 \\
\infty \\
{[69.048-\infty]}\end{array}$ & $\begin{array}{c}52.972 \\
\infty \\
{[47.513-\infty]}\end{array}$ \\
\hline Tea & $\begin{array}{l}\text { LS } \\
\text { Median- } \\
\text { Unbiased }\end{array}$ & $\begin{array}{c}0.926 \\
0.936 \\
{[.906-.967]}\end{array}$ & $\begin{array}{c}0.735 \\
0.768 \\
{[.673-.875]}\end{array}$ & $\begin{array}{c}0.397 \\
0.452 \\
{[.305-.670]}\end{array}$ & $\begin{array}{c}0.063 \\
0.092 \\
{[.028-.301]}\end{array}$ & $\begin{array}{c}13.514 \\
15.625 \\
{[10.618-30.488]}\end{array}$ & $\begin{array}{c}9.016 \\
10.480 \\
{[7.008-20.784]}\end{array}$ \\
\hline $\begin{array}{l}\text { Agricultural } \\
\text { raw materials }\end{array}$ & $\begin{array}{l}\text { LS } \\
\text { Median- } \\
\text { Unbiased }\end{array}$ & $\begin{array}{c}0.964 \\
0.976 \\
{[.954-1.00]}\end{array}$ & $\begin{array}{c}0.864 \\
0.908 \\
{[.827-1.00]}\end{array}$ & $\begin{array}{c}0.644 \\
0.749 \\
{[.565-1.00]}\end{array}$ & $\begin{array}{c}0.267 \\
0.420 \\
{[.180-1.00]}\end{array}$ & $\begin{array}{c}27.778 \\
41.976 \\
{[21.528-\infty]}\end{array}$ & $\begin{array}{c}18.905 \\
28.748 \\
{[14.573-\infty]}\end{array}$ \\
\hline Timber & $\begin{array}{l}\text { LS } \\
\text { Median- } \\
\text { Unbiased }\end{array}$ & $\begin{array}{c}0.944 \\
0.955 \\
{[.928-.984]}\end{array}$ & $\begin{array}{c}0.794 \\
0.831 \\
{[.741-.936]}\end{array}$ & $\begin{array}{c}0.501 \\
0.573 \\
{[.406-.821]}\end{array}$ & $\begin{array}{c}0.126 \\
0.188 \\
{[.067-.554]}\end{array}$ & $\begin{array}{c}17.857 \\
22.077 \\
{[13.832-61.407]}\end{array}$ & $\begin{array}{c}12.028 \\
14.953 \\
{[9.237-42.216]}\end{array}$ \\
\hline Hardwood & $\begin{array}{l}\text { LS } \\
\text { Median- } \\
\text { Unbiased }\end{array}$ & $\begin{array}{c}0.961 \\
1.00 \\
{[.956-1.00]}\end{array}$ & $\begin{array}{c}0.853 \\
1.00 \\
{[.834-1.00]}\end{array}$ & $\begin{array}{c}0.620 \\
1.00 \\
{[.581-1.00]}\end{array}$ & $\begin{array}{c}0.239 \\
1.00 \\
{[.196-1.00]}\end{array}$ & $\begin{array}{c}25.641 \\
\infty \\
{[22.587-\infty]}\end{array}$ & $\begin{array}{c}17.424 \\
\infty \\
{[15.307-\infty]}\end{array}$ \\
\hline $\begin{array}{l}\text { Hardwood } \\
\text { (logs) }\end{array}$ & $\begin{array}{l}\text { LS } \\
\text { Median- } \\
\text { Unbiased }\end{array}$ & $\begin{array}{c}0.960 \\
1.00 \\
{[.954-1.00]}\end{array}$ & $\begin{array}{c}0.849 \\
1.00 \\
{[.830-1.00]}\end{array}$ & $\begin{array}{c}0.613 \\
1.00 \\
{[.572-1.00]}\end{array}$ & $\begin{array}{c}0.230 \\
1.00 \\
{[.187-1.00]}\end{array}$ & $\begin{array}{c}25 \\
\infty \\
{[21.960-\infty]}\end{array}$ & $\begin{array}{c}16.980 \\
\infty \\
{[14.872-\infty]}\end{array}$ \\
\hline $\begin{array}{l}\text { Hardwood } \\
\text { (sawnwood) }\end{array}$ & $\begin{array}{l}\text { LS } \\
\text { Median- } \\
\text { Unbiased }\end{array}$ & $\begin{array}{c}0.955 \\
1.00 \\
{[.946-1.00]}\end{array}$ & $\begin{array}{c}0.832 \\
1.00 \\
{[.801-1.00]}\end{array}$ & $\begin{array}{c}0.575 \\
1.00 \\
{[.514-1.00]}\end{array}$ & $\begin{array}{c}0.191 \\
1.00 \\
{[.136-1.00]}\end{array}$ & $\begin{array}{c}22.222 \\
\infty \\
{[18.549-\infty]}\end{array}$ & $\begin{array}{c}15.054 \\
\infty \\
{[12.507-\infty]}\end{array}$ \\
\hline
\end{tabular}


Table 2. (continued)

\begin{tabular}{|c|c|c|c|c|c|c|c|}
\hline Commodity & Estimator & $\alpha$ & $\operatorname{IR}(4)$ & $\operatorname{IR}(12)$ & $\operatorname{IR}(36)$ & CIR & HLS \\
\hline Softwood & $\begin{array}{l}\text { LS } \\
\text { Median- } \\
\text { Unbiased }\end{array}$ & $\begin{array}{c}0.908 \\
0.950 \\
{[.881-1.00]}\end{array}$ & $\begin{array}{c}0.679 \\
0.814 \\
{[.602-1.00]}\end{array}$ & $\begin{array}{c}0.314 \\
0.540 \\
{[.218-1.00]}\end{array}$ & $\begin{array}{c}0.031 \\
0.158 \\
{[.010-1.00]}\end{array}$ & $\begin{array}{c}10.870 \\
19.990 \\
{[8.396-\infty]}\end{array}$ & $\begin{array}{c}7.182 \\
13.506 \\
{[5.466-\infty]}\end{array}$ \\
\hline $\begin{array}{c}\text { Softwood } \\
(\operatorname{logs})\end{array}$ & $\begin{array}{l}\text { LS } \\
\text { Median- } \\
\text { Unbiased }\end{array}$ & $\begin{array}{c}0.901 \\
0.941 \\
{[.872-1.00]}\end{array}$ & $\begin{array}{c}0.659 \\
0.785 \\
{[.577-1.00]}\end{array}$ & $\begin{array}{c}0.286 \\
0.485 \\
{[.192-1.00]}\end{array}$ & $\begin{array}{c}0.023 \\
0.114 \\
{[.007-1.00]}\end{array}$ & $\begin{array}{c}10.101 \\
17.065 \\
{[7.787-\infty]}\end{array}$ & $\begin{array}{c}6.649 \\
11.479 \\
{[5.043-\infty]}\end{array}$ \\
\hline $\begin{array}{l}\text { Softwood } \\
\text { (sawnwood) }\end{array}$ & $\begin{array}{l}\text { LS } \\
\text { Median- } \\
\text { Unbiased }\end{array}$ & $\begin{array}{c}0.875 \\
0.912 \\
{[.838-1.00]}\end{array}$ & $\begin{array}{c}0.586 \\
0.692 \\
{[.492-1.00]}\end{array}$ & $\begin{array}{c}0.201 \\
0.331 \\
{[.119-1.00]}\end{array}$ & $\begin{array}{c}0.008 \\
0.036 \\
{[.002-1.00]}\end{array}$ & $\begin{array}{c}8 \\
11.351 \\
{[6.159-\infty]}\end{array}$ & $\begin{array}{c}5.191 \\
7.516 \\
{[3.912-\infty]}\end{array}$ \\
\hline Cotton & $\begin{array}{l}\text { LS } \\
\text { Median- } \\
\text { Unbiased }\end{array}$ & $\begin{array}{c}0.979 \\
0.995 \\
{[.973-1.00]}\end{array}$ & $\begin{array}{c}0.919 \\
0.982 \\
{[.898-1.00]}\end{array}$ & $\begin{array}{c}0.775 \\
0.947 \\
{[.724-1.00]}\end{array}$ & $\begin{array}{c}0.466 \\
0.849 \\
{[.380-1.00]}\end{array}$ & $\begin{array}{c}47.619 \\
219.707 \\
{[37.723-\infty]}\end{array}$ & $\begin{array}{c}32.659 \\
151.943 \\
{[25.799-\infty]}\end{array}$ \\
\hline Wool & $\begin{array}{l}\text { LS } \\
\text { Median- } \\
\text { Unbiased }\end{array}$ & $\begin{array}{c}0.978 \\
0.994 \\
{[.972-1.00]}\end{array}$ & $\begin{array}{c}0.915 \\
0.976 \\
{[.893-1.00]}\end{array}$ & $\begin{array}{c}0.766 \\
0.929 \\
{[.711-1.00]}\end{array}$ & $\begin{array}{c}0.449 \\
0.801 \\
{[.360-1.00]}\end{array}$ & $\begin{array}{c}45.455 \\
162.771 \\
{[35.741-\infty]}\end{array}$ & $\begin{array}{c}31.159 \\
112.477 \\
{[24.426-\infty]}\end{array}$ \\
\hline $\begin{array}{l}\text { Wool } \\
\text { (coarse) }\end{array}$ & $\begin{array}{l}\text { LS } \\
\text { Median- } \\
\text { Unbiased }\end{array}$ & $\begin{array}{c}0.976 \\
0.990 \\
{[.969-1.00]}\end{array}$ & $\begin{array}{c}0.907 \\
0.961 \\
{[.881-1.00]}\end{array}$ & $\begin{array}{c}0.747 \\
0.887 \\
{[.683-1.00]}\end{array}$ & $\begin{array}{c}0.417 \\
0.698 \\
{[.319-1.00]}\end{array}$ & $\begin{array}{c}41.667 \\
100.724 \\
{[32.016-\infty]}\end{array}$ & $\begin{array}{c}28.533 \\
69.649 \\
{[21.843-\infty]}\end{array}$ \\
\hline $\begin{array}{l}\text { Wool } \\
\text { (fine) }\end{array}$ & $\begin{array}{l}\text { LS } \\
\text { Median- } \\
\text { Unbiased }\end{array}$ & $\begin{array}{c}0.974 \\
0.988 \\
{[.966-1.00]}\end{array}$ & $\begin{array}{c}0.899 \\
0.952 \\
{[.872-1.00]}\end{array}$ & $\begin{array}{c}0.729 \\
0.864 \\
{[.664-1.00]}\end{array}$ & $\begin{array}{c}0.387 \\
0.645 \\
{[.292-1.00]}\end{array}$ & $\begin{array}{c}38.462 \\
82.639 \\
{[29.767-\infty]}\end{array}$ & $\begin{array}{c}26.311 \\
56.934 \\
{[20.284-\infty]}\end{array}$ \\
\hline Hides & $\begin{array}{l}\text { LS } \\
\text { Median- } \\
\text { Unbiased }\end{array}$ & $\begin{array}{c}0.929 \\
0.939 \\
{[.910-.970]}\end{array}$ & $\begin{array}{c}0.745 \\
0.779 \\
{[.685-.886]}\end{array}$ & $\begin{array}{c}0.413 \\
0.472 \\
{[.321-.695]}\end{array}$ & $\begin{array}{c}0.071 \\
0.105 \\
{[.033-.335]}\end{array}$ & $\begin{array}{c}14.085 \\
16.482 \\
{[11.076-33.428]}\end{array}$ & $\begin{array}{c}9.412 \\
11.074 \\
{[7.325-22.822]}\end{array}$ \\
\hline Rubber & $\begin{array}{l}\text { LS } \\
\text { Median- } \\
\text { Unbiased }\end{array}$ & $\begin{array}{c}0.971 \\
0.984 \\
{[.962-1.00]}\end{array}$ & $\begin{array}{c}0.889 \\
0.937 \\
{[.856-1.00]}\end{array}$ & $\begin{array}{c}0.702 \\
0.823 \\
{[.628-1.00]}\end{array}$ & $\begin{array}{c}0.347 \\
0.557 \\
{[.248-1.00]}\end{array}$ & $\begin{array}{c}34.483 \\
61.956 \\
{[26.298-\infty]}\end{array}$ & $\begin{array}{c}23.553 \\
42.597 \\
{[17.880-\infty]}\end{array}$ \\
\hline Tobacco & $\begin{array}{l}\text { LS } \\
\text { Median- } \\
\text { Unbiased }\end{array}$ & $\begin{array}{c}0.989 \\
1.00 \\
{[.992-1.00]}\end{array}$ & $\begin{array}{c}0.957 \\
1.00 \\
{[.968-1.00]}\end{array}$ & $\begin{array}{c}0.876 \\
1.00 \\
{[.908-1.00]}\end{array}$ & $\begin{array}{c}0.672 \\
1.00 \\
{[.747-1.00]}\end{array}$ & $\begin{array}{c}90.909 \\
\infty \\
{[124.815-\infty]}\end{array}$ & $\begin{array}{c}62.666 \\
\infty \\
{[85.731-\infty]}\end{array}$ \\
\hline Metals & $\begin{array}{l}\text { LS } \\
\text { Median- } \\
\text { Unbiased }\end{array}$ & $\begin{array}{c}0.971 \\
0.984 \\
{[.962-1.00]}\end{array}$ & $\begin{array}{c}0.889 \\
0.936 \\
{[.856-1.00]}\end{array}$ & $\begin{array}{c}0.702 \\
0.821 \\
{[.626-1.00]}\end{array}$ & $\begin{array}{c}0.347 \\
0.552 \\
{[.246-1.00]}\end{array}$ & $\begin{array}{c}34.483 \\
61.167 \\
{[26.141-\infty]}\end{array}$ & $\begin{array}{c}23.553 \\
42.050 \\
{[17.771-\infty]}\end{array}$ \\
\hline Aluminum & $\begin{array}{l}\text { LS } \\
\text { Median- } \\
\text { Unbiased }\end{array}$ & $\begin{array}{c}0.964 \\
0.976 \\
{[.954-1.00]}\end{array}$ & $\begin{array}{c}0.864 \\
0.908 \\
{[.827-1.00]}\end{array}$ & $\begin{array}{c}0.644 \\
0.748 \\
{[.565-1.00]}\end{array}$ & $\begin{array}{c}0.267 \\
0.419 \\
{[.180-1.00]}\end{array}$ & $\begin{array}{c}27.778 \\
41.903 \\
{[21.507-\infty]}\end{array}$ & $\begin{array}{c}18.905 \\
28.697 \\
{[14.558-\infty]}\end{array}$ \\
\hline Copper & $\begin{array}{l}\text { LS } \\
\text { Median- } \\
\text { Unbiased }\end{array}$ & $\begin{array}{c}0.977 \\
0.991 \\
{[.970-1.00]}\end{array}$ & $\begin{array}{c}0.911 \\
0.966 \\
{[.885-1.00]}\end{array}$ & $\begin{array}{c}0.756 \\
0.902 \\
{[.692-1.00]}\end{array}$ & $\begin{array}{c}0.433 \\
0.734 \\
{[.332-1.00]}\end{array}$ & $\begin{array}{c}43.478 \\
116.766 \\
{[33.136-\infty]}\end{array}$ & $\begin{array}{c}29.789 \\
80.589 \\
{[22.620-\infty]}\end{array}$ \\
\hline Iron ore & $\begin{array}{l}\text { LS } \\
\text { Median- } \\
\text { Unbiased }\end{array}$ & $\begin{array}{c}0.966 \\
0.979 \\
{[.956-1.00]}\end{array}$ & $\begin{array}{c}0.871 \\
0.917 \\
{[.836-1.00]}\end{array}$ & $\begin{array}{c}0.660 \\
0.771 \\
{[.584-1.00]}\end{array}$ & $\begin{array}{c}0.288 \\
0.459 \\
{[.199-1.00]}\end{array}$ & $\begin{array}{c}29.412 \\
46.721 \\
{[22.832-\infty]}\end{array}$ & $\begin{array}{c}20.038 \\
32.037 \\
{[15.477-\infty]}\end{array}$ \\
\hline Lead & $\begin{array}{l}\text { LS } \\
\text { Median- } \\
\text { Unbiased }\end{array}$ & $\begin{array}{c}0.975 \\
0.989 \\
{[.968-1.00]}\end{array}$ & $\begin{array}{c}0.904 \\
0.957 \\
{[.877-1.00]}\end{array}$ & $\begin{array}{c}0.738 \\
0.878 \\
{[.675-1.00]}\end{array}$ & $\begin{array}{c}0.402 \\
0.676 \\
{[.308-1.00]}\end{array}$ & $\begin{array}{l}40 \\
92.400 \\
{[31.063-\infty]}\end{array}$ & $\begin{array}{c}27.378 \\
63.699 \\
{[21.183-\infty]}\end{array}$ \\
\hline Nickel & $\begin{array}{l}\text { LS } \\
\text { Median- } \\
\text { Unbiased }\end{array}$ & $\begin{array}{c}0.980 \\
0.996 \\
{[.974-1.00]}\end{array}$ & $\begin{array}{c}0.922 \\
0.984 \\
{[.900-.984]}\end{array}$ & $\begin{array}{c}0.785 \\
0.954 \\
{[.729-.954]}\end{array}$ & $\begin{array}{c}0.483 \\
0.867 \\
{[.338-.867]}\end{array}$ & $\begin{array}{c}50 \\
253.251 \\
{[38.532-\infty]}\end{array}$ & $\begin{array}{c}34.310 \\
175.193 \\
{[26.360-\infty]}\end{array}$ \\
\hline Tin & $\begin{array}{l}\text { LS } \\
\text { Median- } \\
\text { Unbiased }\end{array}$ & $\begin{array}{c}0.949 \\
0.807 \\
{[.679-.886]}\end{array}$ & $\begin{array}{c}0.811 \\
0.424 \\
{[.212-.616]}\end{array}$ & $\begin{array}{c}0.534 \\
0.076 \\
{[.010-.234]}\end{array}$ & $\begin{array}{c}0.151 \\
0.000 \\
{[.000-.013]}\end{array}$ & $\begin{array}{c}19.608 \\
5.181 \\
{[3.115-8.772]}\end{array}$ & $\begin{array}{c}13.241 \\
3.232 \\
{[1.791-5.727]}\end{array}$ \\
\hline
\end{tabular}




\section{Table 2. (concluded)}

\begin{tabular}{|c|c|c|c|c|c|c|c|}
\hline Commodity & Estimator & $\alpha$ & $\operatorname{IR}(4)$ & $\operatorname{IR}(12)$ & $\operatorname{IR}(36)$ & CIR & HLS \\
\hline Zinc & $\begin{array}{l}\text { LS } \\
\text { Median- } \\
\text { Unbiased }\end{array}$ & $\begin{array}{c}0.978 \\
0.993 \\
{[.971-1.00]}\end{array}$ & $\begin{array}{c}0.915 \\
0.971 \\
{[.889-1.00]}\end{array}$ & $\begin{array}{c}0.766 \\
0.916 \\
{[.702-1.00]}\end{array}$ & $\begin{array}{c}0.449 \\
0.768 \\
{[.346-1.00]}\end{array}$ & $\begin{array}{c}45.455 \\
136.664 \\
{[34.409-\infty]}\end{array}$ & $\begin{array}{c}31.159 \\
94.381 \\
{[23.502-\infty]}\end{array}$ \\
\hline Fertilizer & $\begin{array}{l}\text { LS } \\
\text { Median- } \\
\text { Unbiased }\end{array}$ & $\begin{array}{c}0.972 \\
0.985 \\
{[.964-1.00]}\end{array}$ & $\begin{array}{c}0.893 \\
0.943 \\
{[.862-1.00]}\end{array}$ & $\begin{array}{c}0.711 \\
0.838 \\
{[.641-1.00]}\end{array}$ & $\begin{array}{c}0.360 \\
0.589 \\
{[.264-1.00]}\end{array}$ & $\begin{array}{c}35.714 \\
68.405 \\
{[27.506-\infty]}\end{array}$ & $\begin{array}{c}24.407 \\
47.067 \\
{[18.717-\infty]}\end{array}$ \\
\hline $\begin{array}{l}\text { Phosphate } \\
\text { rock }\end{array}$ & $\begin{array}{l}\text { LS } \\
\text { Median- } \\
\text { Unbiased }\end{array}$ & $\begin{array}{c}0.972 \\
0.986 \\
{[.964-1.00]}\end{array}$ & $\begin{array}{c}0.893 \\
0.944 \\
{[.864-.944]}\end{array}$ & $\begin{array}{c}0.711 \\
0.843 \\
{[.645-.843]}\end{array}$ & $\begin{array}{c}0.360 \\
0.598 \\
{[.268-.598]}\end{array}$ & $\begin{array}{c}35.714 \\
70.527 \\
{[27.786-\infty]}\end{array}$ & $\begin{array}{c}24.407 \\
48.538 \\
{[18.973-\infty]}\end{array}$ \\
\hline $\begin{array}{l}\text { Triple super- } \\
\text { phosphate }\end{array}$ & $\begin{array}{l}\text { LS } \\
\text { Median- } \\
\text { Unbiased }\end{array}$ & $\begin{array}{c}0.985 \\
1.00 \\
{[.983-1.00]}\end{array}$ & $\begin{array}{c}0.941 \\
1.00 \\
{[.934-1.00]}\end{array}$ & $\begin{array}{c}0.834 \\
1.00 \\
{[.814-1.00]}\end{array}$ & $\begin{array}{c}0.580 \\
1.00 \\
{[.540-1.00]}\end{array}$ & $\begin{array}{c}66.667 \\
\infty \\
{[58.920-\infty]}\end{array}$ & $\begin{array}{c}45.862 \\
\infty \\
{[40.492-\infty]}\end{array}$ \\
\hline Petroleum (crude) & $\begin{array}{l}\text { LS } \\
\text { Median- } \\
\text { Unbiased }\end{array}$ & $\begin{array}{c}0.991 \\
1.00 \\
{[.992-1.00]}\end{array}$ & $\begin{array}{c}0.964 \\
1.00 \\
{[.969-1.00]}\end{array}$ & $\begin{array}{c}0.897 \\
1.00 \\
{[.909-1.00]}\end{array}$ & $\begin{array}{c}0.722 \\
1.00 \\
{[.751-1.00]}\end{array}$ & $\begin{array}{c}111.111 \\
\infty \\
{[126.502-\infty]}\end{array}$ & $\begin{array}{c}76.669 \\
\infty \\
{[87.337-\infty]}\end{array}$ \\
\hline Gasoline* & $\begin{array}{l}\text { LS } \\
\text { Median- } \\
\text { Unbiased }\end{array}$ & $\begin{array}{c}0.934 \\
0.984 \\
{[.916-1.00]}\end{array}$ & $\begin{array}{c}0.761 \\
0.939 \\
{[.703-1.00]}\end{array}$ & $\begin{array}{c}0.441 \\
0.829 \\
{[.347-1.00]}\end{array}$ & $\begin{array}{c}0.086 \\
0.570 \\
{[.042-1.00]}\end{array}$ & $\begin{array}{c}15.152 \\
64.475 \\
{[11.844-\infty]}\end{array}$ & $\begin{array}{c}10.152 \\
44.343 \\
{[7.858-\infty]}\end{array}$ \\
\hline Gold* & $\begin{array}{l}\text { LS } \\
\text { Median- } \\
\text { Unbiased }\end{array}$ & $\begin{array}{c}0.995 \\
1.00 \\
{[.999-1.00]}\end{array}$ & $\begin{array}{c}0.980 \\
1.00 \\
{[.997-1.00]}\end{array}$ & $\begin{array}{c}0.942 \\
1.00 \\
{[.990-1.00]}\end{array}$ & $\begin{array}{c}0.835 \\
1.00 \\
{[.972-1.00]}\end{array}$ & $\begin{array}{l}200 \\
\infty \\
{[1250-\infty]}\end{array}$ & $\begin{array}{c}138.283 \\
\infty \\
{[866.222--\infty]}\end{array}$ \\
\hline Heating oil* & $\begin{array}{l}\text { LS } \\
\text { Median- } \\
\text { Unbiased }\end{array}$ & $\begin{array}{c}0.880 \\
0.917 \\
{[.844-1.00]}\end{array}$ & $\begin{array}{c}0.599 \\
0.708 \\
{[.508-1.00]}\end{array}$ & $\begin{array}{c}0.216 \\
0.356 \\
{[.131-1.00]}\end{array}$ & $\begin{array}{c}0.010 \\
0.045 \\
{[.002-1.00]}\end{array}$ & $\begin{array}{c}8.333 \\
12.114 \\
{[6.415-\infty]}\end{array}$ & $\begin{array}{c}5.422 \\
8.045 \\
{[4.090-\infty]}\end{array}$ \\
\hline Natural gas* & $\begin{array}{l}\text { LS } \\
\text { Median- } \\
\text { Unbiased }\end{array}$ & $\begin{array}{c}0.965 \\
1.00 \\
{[.962-1.00]}\end{array}$ & $\begin{array}{c}0.867 \\
1.00 \\
{[.858-1.00]}\end{array}$ & $\begin{array}{c}0.652 \\
1.00 \\
{[.631-1.00]}\end{array}$ & $\begin{array}{c}0.277 \\
1.00 \\
{[.251-1.00]}\end{array}$ & $\begin{array}{c}28.571 \\
\infty \\
{[26.534-\infty]}\end{array}$ & $\begin{array}{c}19.456 \\
\infty \\
{[18.043-\infty]}\end{array}$ \\
\hline
\end{tabular}

Source: Authors' calculations.

Notes: The results of this table are based on Andrews (1993) — see equation (1). IR $(h)$ denotes the impulse response function at time horizon $h$ (see equation (3)). CIR denotes the cumulative impulse response. HLS is, for the autoregressive parameter $\alpha \geq 0$, the length of time (in months) until the impulse response of a unit shock to an economic time series is half its initial magnitude (see equation (4)). The entries in the rows labeled LS are the estimates of $\alpha, \operatorname{IR}(h)$, CIR and HLS obtained using the least squares estimates of $\alpha$ from the regression equation (1). The entries in the rows labeled median-unbiased are the median-unbiased estimates of $\hat{\alpha}, I \hat{R}(h), C \hat{I} R$ and $H \hat{L} S$ of $\alpha, \operatorname{IR}(h)$, CIR, and HLS, respectively, as defined in Section I. The intervals in square brackets below the median-unbiased estimates are the 90 percent confidence intervals. The median-unbiased estimates and exact confidence intervals given in this table were determined using quantiles generated by Monte Carlo simulation (involving 10,000 replications) and interpolation for sample sizes not given in Andrews (1993) or in Appendix III of this paper.

Those commodities denoted by bold and an asterisk are not weighted in the all commodities index (for example, natural gas*); those commodities indented and denoted by bold are components of the all commodities index (for example, nonfuel and crude petroleum); those commodities indented and denoted by bold and italics are components of the nonfuel commodities index (for example, food); those commodities indented and denoted by italics are subcomponents of the components of the nonfuel commodities index, which comprise those that are aggregated from more than one individual commodity index (for example, cereals) and individual commodity indices (for example, tea); those commodities indented are the individual indices of the subcomponents of the nonfuel commodities index (for example, maize). For a description of the commodity price series, and their weights in the aggregate indices, see Appendix I. For all variables, the data are monthly over the sample period 1957:1 to 1998:12, except for the following price indices which all end at 1998:12 but begin at the following dates: gasoline (1982:6); gold (1964:1); hardwood (1982:5); hardwood (logs, 1982:5); hardwood (sawn, 1982:5); heating oil (1986:6); natural gas (1985:1); softwood (1982:5); softwood (logs, 1982:5); softwood (sawn, 1982:5); and tobacco (1967:12). 
MU estimate. Accordingly, shocks to coarse wool do appear to be rather persistent, and given that the median-unbiased measure of HLS for coarse wool lies outside the 90 percent confidence interval for the estimated HLS for tea, shocks to coarse wool are significantly longer than those affecting tea. ${ }^{12}$

For tobacco the median-unbiased estimate of $\alpha$ is 1.00 , as opposed to the least squares estimate of 0.989 . As for tea, while the difference between these two estimates is small in absolute terms, it has a large effect on calculations of the persistence of the time series. The length of the 90 percent confidence interval for the median-unbiased estimate of $\alpha(0.992,1.00)$ indicates that $\alpha$ is estimated very precisely. Also, the IRF for the MU estimate do not decline monotonically toward zero. The estimates yield an infinite [LS 90.989, MU $\infty$ ] increase in the estimate of CIR (the total cumulative effect of a unit shock on the future evolution of the price series). This indicates that, for example, a unit shock to tobacco generates an eventual $(t=\infty)$ infinite displacement of the level of the price of tobacco-the effect of a unit shock becomes a permanent component of the time series. Moreover, the time it takes for half of the impulse response of a shock to the price of tobacco to dissipate is 62.666 months for the LS estimate, while the MU estimate indicates that price shocks do not dissipate. Accordingly, shocks to tobacco are permanent, and so tobacco prices do not display mean-reverting behavior in response to a shock to prices. The MU estimates of HLS and the associated confidence intervals indicate that there is much better evidence in favor of very longlived shocks for tobacco than for coarse wool.

In general, the median-unbiased estimates of $\alpha$ are closer to 1.00 than the least squares estimates, with the difference between them (a measure of the downward bias of the least squares estimator) ranging between 0.004 and 0.05 (see Table 2). In turn, these differences generate median-unbiased estimates of the CIR and HLS that are between near zero and 326 percent larger than those generated from the least squares estimates. Bananas have the lowest estimate of $\alpha$ (of 0.748). The 90 percent confidence intervals for most series range between 0.950 and 1.00; the confidence interval for other series differs somewhat, ranging in length from 0.001 (gold) to 0.156 (heating oil).

Of interest is the persistence results for the 8 aggregated series, in comparison with the results for the 44 individual commodity series. Shocks to the all commodities, nonfuel, beverages, and food series do not dissipate, indicating that shocks to these aggregated commodity series are permanent. ${ }^{13}$ For these series, the current price can be expressed as the price in the previous period, plus a random shock; accordingly, currently low prices will tend to remain low in subsequent

\footnotetext{
${ }^{12}$ The difference between the HLS and CIR for any two given commodities is statistically significant (at the 5 percent level) if the confidence interval for the HLS and CIR of the first commodity lies outside the confidence interval for the HLS and CIR of the second commodity. For example, the difference in the median-unbiased estimates of CIR and HLS between tea (15.625 and 10.480, respectively) and coarse wool (100.724 and 69.649, respectively) is statistically significant, as the confidence intervals for tea's CIR and HLS (10.618-30.488 and 7.008-20.784, respectively) do not overlap with the confidence inter-

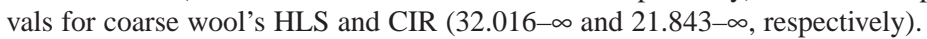

${ }^{13}$ This result is perhaps not surprising, given that these aggregated indices are formed from some individual commodity series that themselves have a unit root.
} 
periods, and will not tend to revert to an average level. In contrast, the aggregated series for agricultural raw materials, fertilizer, metals, and sugar exhibit relatively short half-lives. For these series, prices do eventually revert to their average values. Accordingly, it is important to examine the time series properties of individual commodities before making judgments about the persistence of shocks affecting them, as the persistence of shocks to the aggregated series may differ greatly from the persistence of shocks to individual time series.

The above results indicate that, using the median-unbiased model, the majority of the 44 individual commodity series are subject to price shocks that are not permanent (that is, they are less than infinitely persistent). Given that most price shocks have their origin on the supply side of the market, we typically find that those commodities with less persistent shocks are those where the weather influences the generation of supply shocks. Those commodities with more persistent shocks tend to be those where weather plays a limited role. For persistent shocks it is likely that the entry of new producers could have long-run effects on the evolution of prices (metals, fuels, and fertilizers), and that those commodities where shocks have a greater possibility of originating from the demand side of the market could result in long periods of excess supply or demand (such as in the development of synthetic substitutes (wool and cotton), or technological change in demand for inputs (metals)). Persistent shocks could also arise owing to the formation or termination of international commodity price agreements (Gersovitz and Paxson, 1990). The strong showing of beverages in the persistence of price shocks is most likely due to supply shocks that have long-lasting effects on prices for tree crops (such as frosts, fires, and diseases). For both beverages and metals, high prices induce investment activity that, because of long periods of gestation, can result in extended periods of excess productive capacity and flat prices. ${ }^{14}$

An alternative method of calculating persistence to that derived here is the nonparametric estimator of Cochrane (1988), which measures the fraction of a price shock that can be expected to persist into the indefinite future. Gersovitz and Paxson (1990) apply Cochrane's method to estimate persistence, using annual World Bank commodity data for the period 1950-87. They find persistence measures that are typically lower than would be suggested by low-order ARMA models. ${ }^{15}$ Cochrane's (1988) approach does provide information as to whether the volatility of commodity price shocks is mostly in the short run or mostly in the long run, but such information is of limited use to policymakers in determining how to respond to shocks. The key question for policymakers concerns the typical duration of price shocks to commodities; that is, whether shocks are typically

\footnotetext{
${ }^{14}$ The duration of commodity-price shocks varies greatly across commodities (Cashin, McDermott, and Scott, 1999). For example, the beverage boom of the late 1970s (driven largely by adverse weather conditions during a period of relatively low production) resulted in price booms for cocoa and arabica coffee which lasted about 25 months. In contrast, the price of gold endured a decade-long slump in the low-inflation environment of the 1980s, apart from a short-lived boom in prices between mid-1986 and mid-1987.

15Deaton and Laroque (1992) and León and Soto (1997) also implement Cochrane's (1988) method, using the Grilli-Yang (1988) annual data. They find that the majority of commodity price series exhibit shocks which eventually revert to their mean or to a deterministic trend.
} 
short-lived (in which case scope may exist for price stabilization or consumptionsmoothing policies) or typically long-lived (in which case structural adjustment to the new equilibrium is warranted). In contrast to Cochrane (1988), the Andrews (1993) approach used in this paper to measure persistence does enable the calculation of unbiased scalar measures of the duration and variability of typical price shocks.

It is well known that unit root tests have difficulty in distinguishing between nonstationarity and stationarity with a structural break (Perron, 1989). Moreover, autoregressive models with (endogenous or exogenous) structural change impart further bias to the autoregressive coefficient and diminish the power of standard unit root tests. Our analysis of the persistence of shocks has been predicated on the absence of structural breaks that might impart persistence to the evolution of the commodity price series. For the stationary price series (34 of the 44 individual commodities analyzed in this paper), McDermott (1994) has shown that the median-unbiased persistence measure is invariant to the presence of a break. However, for the nonstationary price series subject to permanent shocks (10 of the 44 commodities), not accounting for structural breaks may matter, as for these series we may have erroneously rejected a finding of finite persistence (with a break) in favor of a finding of permanent shocks. Accordingly, we implement the Zivot-Andrews (1992) test for structural breaks, and no breaks are discovered for 9 of the 10 series found to have permanent shocks. ${ }^{16}$ Only one series, tin, is found to be trend stationary with a structural break (at 1985:10), which coincides with the dramatic collapse of the International Tin Agreement on October 24, 1985. ${ }^{17}$

While it is important to ascertain the duration of shocks to commodity prices, knowledge of the size of price shocks is also of great interest. Very small, yet longlived, price shocks will have different implications for the appropriateness of any policy response (particularly the efficacy of price stabilization schemes) than very large, long-lived price shocks. In this connection, our estimation of persistence enables a calculation of the size of shocks to commodity prices. For example, in the case of petroleum (spot crude), the standard error of the regression (equation (1)) is 0.0827 , indicating that one-third of the time, the market for petroleum will be faced with the prospect of a price change of greater than 8 percent. Using the specific case of oil-dependent Nigeria as an example, in 1998 Nigerian petroleum traded at about US\$12.80 per barrel. Accordingly, in any given month there is a one-in-six chance that the realized price of petroleum would drop by about US\$1.06 per barrel or more. Nigerian oil exports totaled about 2 million barrels

${ }^{16}$ The Zivot-Andrews (1992) sequential unit root test, allowing for a structural change (level shift) at an unknown (endogenous) breakpoint, yields a test statistic of -5.84 , which is smaller than the critical value (at the 5 percent level of significance) of -4.80 , indicating rejection of the null hypothesis of a unit root in the case of tin.

${ }^{17}$ In examining tin, we can measure the extent to which not accounting for a structural break has biased the estimate of the autoregressive parameter $(\alpha)$ toward one. The value of the estimated $\alpha$ for tin arising from the least squares regression (including an intercept and time trend) at the point of the structural break (1985:10) is 0.949. Using the results provided in McDermott (1994, Table 3.1) yields an asymptotically median-unbiased estimate of $\alpha=0.807$ and a 90 percent confidence interval of $(0.679$, 0.886). This corrected measure of $\alpha$ for tin has been used in the calculations contained in Table 2 . 
per day in 1998, and so, given our findings as to the permanence of shocks to oil, there is a one-in-six chance that such an adverse shock may occur, which would result in a reduction of its petroleum export revenues by about 8 percent (about US\$750 million per year in 1998 dollars) in perpetuity (see Tables 1-2, and IMF, 1999). Clearly, not only are shocks to petroleum very long-lived, but they can also be large in magnitude.

Finally, the higher-order moments of the price series will also affect the feasibility of stabilization policies. If price shocks are skewed toward the lower tail of the distribution (left skewness), then it is likely there is a downward trend in prices for this commodity, implying that stabilization is likely to be more costly than if there was no trend in the series or if its distribution was skewed toward positive shocks. Similarly, if there is excess kurtosis, then big shocks are relatively frequent events. Even if such shocks are temporary, any stabilization scheme would require extremely large funds to effectively stabilize producer returns. As noted in Section II, the prices of all commodities display significant leptokurtosis, and most commodity-price series display significant skewness. For example, iron ore prices exhibit significant left skewness, while cocoa prices exhibit significant right skewness (see Cashin, McDermott, and Scott, 1999).

\section{Policy Issues}

Variability in the prices of major commodity exports contributes greatly to variability in the export revenues of commodity-dependent countries. When there is a fall in the real price of a commodity-exporting country's dominant export, then it experiences a decline in the international purchasing power of a given basket of its national output. Clearly, both short-run volatility and long-run trend movements in commodity prices present serious challenges for many developing countries, because of their large impacts on both the balance of payments and government budgetary positions. In the design of an appropriate policy response, it is important to have knowledge of the typical duration of shocks to commodity prices. For example, a key input to the composition, sequencing, and duration of structural adjustment programs for countries suffering depressed world prices for their important exports will be expectations as to whether export returns will bounce back quickly or instead are expected to continue to be depressed for a long period. In addition, the efficacy and efficiency of government commodity stabilization schemes will be dependent on whether price shocks to major exports are expected to be short-lived or long-lasting.

\section{Schemes to Ameliorate Price Variability}

Variable commodity prices may result not only in variable export revenues, but also in variable producer surpluses, variable patterns of consumption, and variability in government fiscal positions. While borrowing and lending can in principle offset the impact on consumers, producers, and government, in many developing countries poorly functioning financial systems and sovereign risk problems limit their recourse to domestic and external financing as a response to 
price variability. Instead, the attention of policymakers has been largely focused on the need to stabilize commodity export earnings, and one or more of the following measures have typically been adopted: ${ }^{18}$

(i) stabilization of world commodity prices (through the exercise of market power by a monopolistic producer or producer cartel, or through international commodity agreements involving both importing and exporting countries); (ii) stabilization of producer revenues, given producer prices (using risk management instruments such as swaps and forward, futures and options contracts); (iii) stabilization of government revenues, given world commodity prices (by the implementation of revenue stabilization funds, designed to undertake precautionary savings to absorb cyclical variation in export revenues and profits from state utilities); (iv) compensatory financing to offset variability in export revenues, given world commodity prices (through domestic stabilization funds or international schemes for compensatory finance); and (v) stabilization of domestic producer and/or consumer prices, given world prices (through a variable export tax or tariff, the activities of agricultural marketing boards, or the operation of domestic stockpiles and stabilization funds).

As our interest in this paper is not with the policy implications of stabilizing producer ${ }^{19}$ or government revenues, ${ }^{20}$ we will concentrate on the implications of our persistence analysis for policy issues in the remaining aspects of public intervention to stabilize commodity price shocks. It should also be recognized that apart from the consumption-smoothing arguments for stabilization schemes (which essentially stand or fall on the persistence of shocks), there may be riskreduction arguments for such schemes. We will not be directly discussing the policy response to price volatility in this paper-a discussion of this can be found in Reinhart and Wickham (1994) and Borensztein and Reinhart (1994), among others.

Traditionally, governments in developing countries have assumed a major role in smoothing the domestic effects of fluctuations in world commodity prices, typically by stockholding activities designed to curtail price variability. The rationales for government intervention are usually based on market imperfections, such as financial market imperfections and information problems (Deaton, 1992). These policy actions often set price stabilization as a major objective, and at the domestic level are typically implemented through institutional arrangements such as agricultural marketing boards and stabilization funds. At the international level, commodity agreements and compensatory financing from international organizations (such as the International Monetary Fund's Contingency and Compensatory Financing Facility (CCFF)) have also been used to smooth the effects on export

\footnotetext{
18This categorization is adapted from Gilbert (1993).

${ }^{19}$ For a description of the scope for private agents in commodity-exporting countries to use marketbased risk management instruments (such as futures, swaps, and options) as alternatives to national and international commodity stabilization schemes, see World Bank (1994).

${ }^{20}$ An example of a revenue-stabilization mechanism is Chile's Copper Stabilization Fund. The fund, established in 1985, uses foreign exchange reserves as a buffer against the variability of export tax receipts, with the goal of smoothing the effect of copper price changes on the variability of public revenues received from the state copper utility.
} 
earnings of shocks to world commodity prices. Aside from the issue of whether the government is better at managing commodity price shocks than private agents, the success of such intervention strategies critically hinges on the assumption that the nature of the commodity price shock is temporary, and accordingly is expected to reverse itself in the short-run. Using the results presented in Section III, we examine these issues further below.

\section{Differences in the Persistence of Shocks to Commodity Prices}

The findings of this study suggest that shocks to most commodity prices are typically finite in duration, with 44 of the 60 aggregated and individual series having finite median HLS measures (Table 2). Were we to follow the standard practice in the literature, we would then most likely conclude that for these price series, scope did exist for commodity stabilization schemes and external borrowing to smooth the path of national consumption (León and Soto, 1997).

However, the scalar measures of persistence derived from the Andrews (1993) method indicate that for many of the commodity price series exhibiting finitelypersistent shocks, the mean reversion of prices typically takes an extremely long time. If we arbitrarily choose a generous HLS of five years (60 months) as the cutoff point beyond which the cost (involving storage, financing, and outputreduction costs) of maintaining any stabilization scheme would be likely to become prohibitive, then 17 of the 44 individual commodity price series are in excess of this cutoff, yet still have finite persistence (see third and fourth columns of Table 3 and Figures 5c-5d). For these 17 series, it is doubtful that the consumption-smoothing gains arising from price stabilization would exceed the cost of maintaining the stabilization agreement. In fact, we find that the persistence of price shocks (measured in terms of HLS) is less than 5 years (60 months) for only 18 of the 44 individual commodity price series (see Table 3 and Figures 5a-5b). Column one of Table 3 and Figure 5a reveal that commodity stabilization schemes are on firmest ground, in terms of persistence of shocks lasting less than one year, for just 8 of the 44 individual commodity price series. ${ }^{21}$

In interpreting Table 3, it should also be kept in mind that the actual HLS for any given commodity has a 50 percent chance of being lower than the estimated median HLS and a 50 percent chance of exceeding the estimated median HLS. For example, while the estimated median HLS for rubber is 43 months (Tables 2-3 and Figure 5b), half of the actual realizations of the HLS for rubber will be less than, and half will exceed, the estimated median HLS. If the maximum length for

\footnotetext{
${ }^{21}$ One cautionary note is that there could be seasonal (chiefly demand-based) effects associated with several of the commodities yielding short-lived measures of persistence (Tables 2 and 3 ). The presence of seasonality effects would be expected to bias downwards the median-unbiased $\hat{\alpha}$ and, accordingly, associated scalar measures of persistence. However, unit root regressions using lags of three, six, and nine months failed to find any large differences in the calculated half-lives of the shocks for those commodities where seasonality was suspected (bananas, tea, heating oil, and hides). For example, the estimated median HLS for bananas using the monthly data is 2.392 months (Table 2); the other half-life calculations were: for three lags (0.766 quarters, that is 2.298 months), six lags (0.556 half-years, 3.336 months) and nine lags (0.681 three-quarter years, 6.129 months).
} 


\section{Table 3. Persistence of Price Shocks (HLS), Individual Commodities,} 1957:1-1998:12

$\begin{array}{lllll}\text { Less Than 1 Year } & \text { 1-4 Years } & \text { 5-8 Years } & 9-18 \text { Years } & \infty \\ \text { Bananas } & \text { Aluminum } & \text { Beef } & \text { Coffee (other milds) } & \text { Cocoa beans } \\ \text { Heating oil } & \text { Fish meal } & \text { Coconut oil } & \text { Cotton } & \text { Coffee (robusta) } \\ \text { Hides } & \text { Gasoline } & \text { Copper } & \text { Nickel } & \text { Gold } \\ \text { Softwood (logs) } & \text { Iron ore } & \text { Groundnut oil } & \text { Sugar (free market) } & \text { Hardwood (logs) } \\ \text { Softwood (sawnwood) } & \text { Lamb } & \text { Lead } & \text { Rice } & \text { Natural gas } \\ \text { Sugar (EU) } & \text { Rubber } & \text { Maize } & & \text { Petroleum (crude) } \\ \text { Tea } & \text { Soybean meal } & \text { Palm oil } & & \text { Hardwood (sawnwood) } \\ \text { Tin } & \text { Soybean } & \text { Phosphate rock } & & \text { Tobacco } \\ & \text { Sugar (U.S.) } & \text { Soybean oil } & & \text { Triple superphosphate } \\ & \text { Wheat } & \text { Wool (coarse) } & & \\ & & \text { Wool (fine) } & & \end{array}$

Source: Authors' calculations, derived using equation (4) and the median-unbiased estimates of the autoregressive parameter $(\alpha)$ set out in Table 2 .

Notes: HLS is, for $\alpha \geq 0$, the length of time until the impulse response of a unit shock to an economic time series is half its initial magnitude (see equation (4)). For a description of the commodity price series, see Appendix I.

the sustainability of any rubber stabilization scheme was a HLS of 5 years (60 months), then policymakers would need to factor in the likelihood that actual shocks to rubber prices could last longer than this, notwithstanding that the estimated median is within the sustainable period of time. In contrast, if the maximum length for the sustainability of any tea stabilization scheme was also a HLS of 5 years (60 months), then policymakers might feel more comfortable implementing such a scheme for tea rather than for rubber, given that the estimated median HLS for tea is only 10 months, which is much further from the maximum length of financial sustainability. ${ }^{22}$

As set out in Table 3 (column five) and Figure 5e, for 9 of the 44 individual commodity price series, we find that their price shocks are typically very long-lived (permanent). If commodity price shocks are highly persistent, then national or international arrangements to smooth price shocks will not be sustainable, and countries dependent on international trade in commodities affected by these long-lived shocks will need to adjust their macroeconomic and structural policies to conform with their new steady-state levels of national income, consumption, and wealth.

By providing median-unbiased measures of persistence and their associated confidence intervals, the Andrews (1993) approach allows policymakers to determine their own particular level of tolerance of the risk of the realization of a highly persistent shock to their commodity of interest. Using the HLS as our measure of persistence, and again taking the examples of tea and rubber, respectively, we find

${ }^{22}$ Although tea, like cocoa and coffee, is a tree crop with a gestation lag of three to four years before new plants bear yields, unlike other tree crops, output can be significantly increased in the short run from existing capacity by the practice of "coarse plucking," which involves taking more leaves than normal, at the expense of future yields and some quality (IMF, 1986). 
Figure 5. Results of Median-Unbiased Autoregressive/Unit Root Estimation, Median and 90 Percent Confidence Level of HLS, Individual Commodities, 1957:1-1998:12

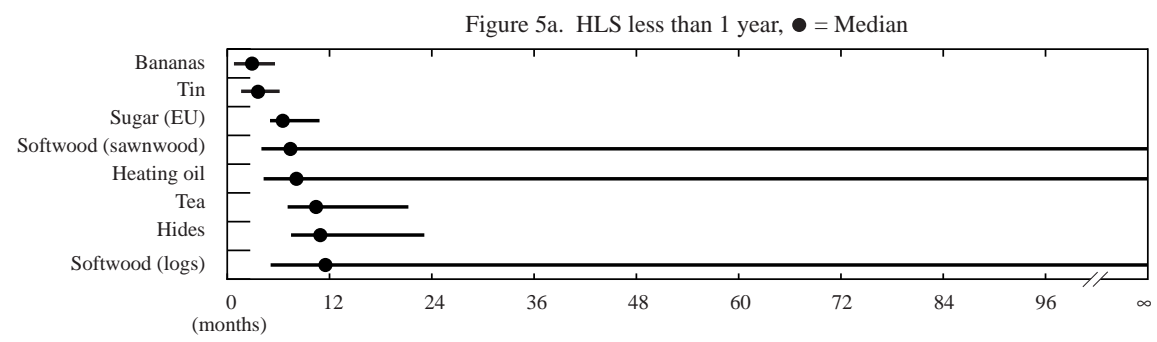

Figure 5b. HLS $1-4$ years, $\bullet=$ Median

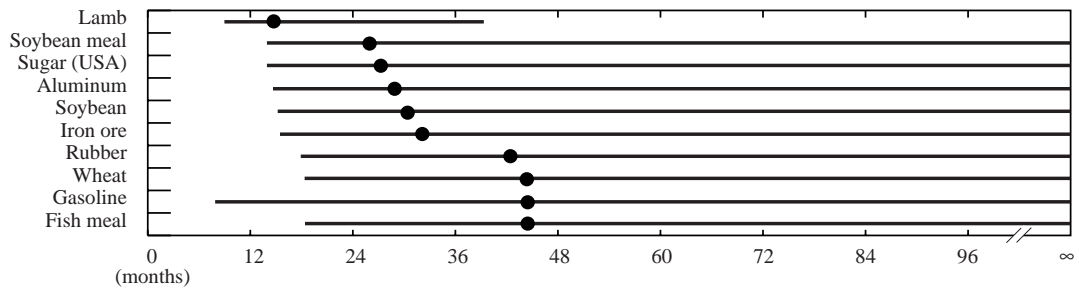

Figure 5c. HLS $5-8$ years, $\bullet=$ Median

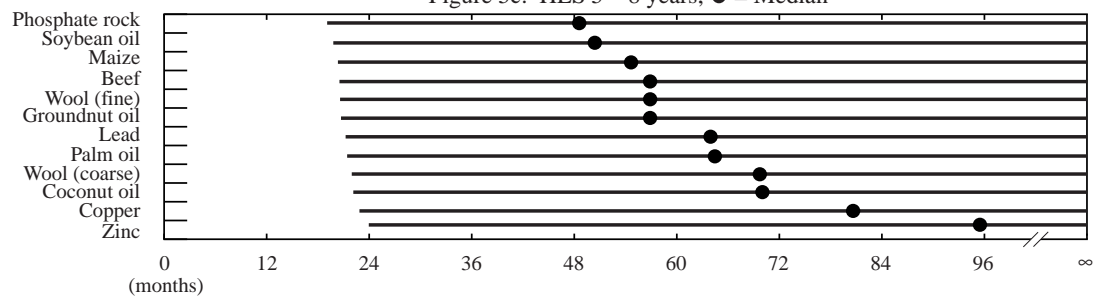

Figure 5d. HLS $9-18$ years, $\bullet=$ Median

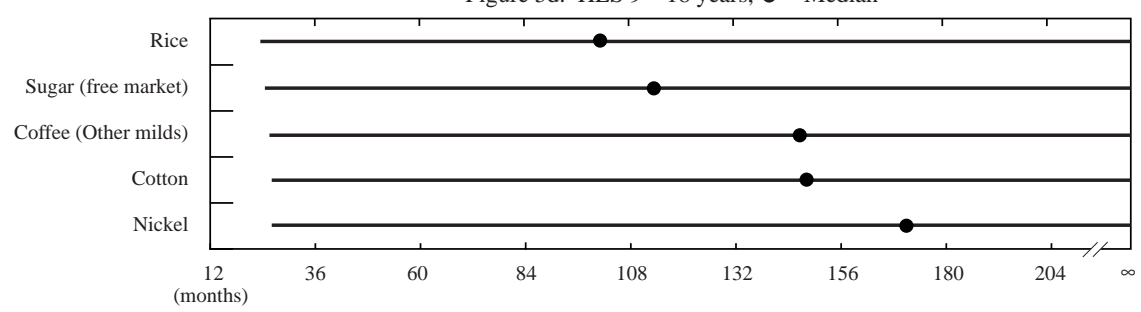

Figure 5e. HLS $\infty, \bullet=$ Median

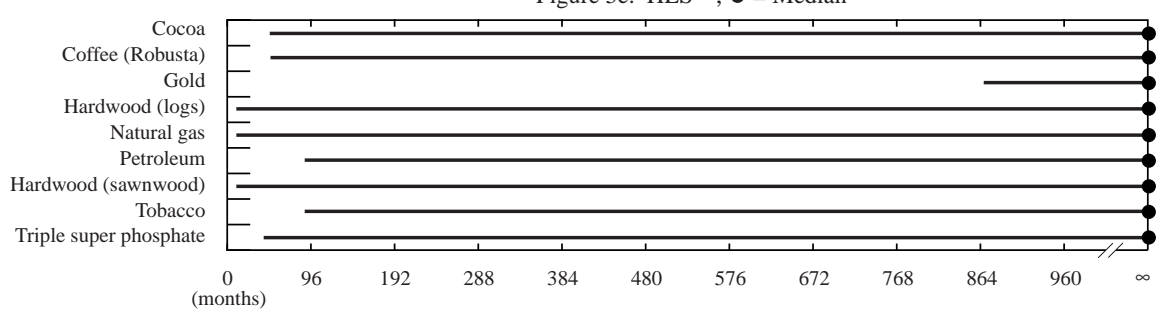

Source: Authors' calculations. The median HLS is denoted by the circle, while the 90 percent confidence interval for the median HLS is denoted by the range of the bar. These results match the median-unbiased results of the last column of Table 2. See Table 2 for additional details and Appendix I for a description of the commodity price series. 
that the estimated 90 percent confidence intervals range from 7 to 21 months and from 18 months to infinity. Accordingly, 5 percent of the actual realizations of the HLS for tea will exceed 21 months, while 5 percent (or more) of the actual realizations of the HLS for rubber will be permanent. This implies that a stabilization scheme for tea is much less likely to collapse than one established for rubber. If we look at all of the 44 individual commodity price series, and again assume that the maximum length for the sustainability of any stabilization scheme is a HLS of 60 months, then there are only six commodities (tea, tin, sugar (EU), bananas, hides, and lamb) for which 95 out of 100 realizations of the HLS will be within this sustainability limit (Tables 2-3 and Figures 5a-5b). While these results do not entirely rule out the successful operation of stabilization schemes for those commodities outside the six mentioned above, they do highlight the much greater likelihood that such schemes will confront shocks which last longer than the maximum sustainable length of time, and thus may not be financially sustainable. ${ }^{23}$

In addition, while our analysis does not differentiate between positive and negative shocks to commodity prices, it is clear that in the actual commodity price data upward movements in prices are typically shorter in duration than downward movements in prices (Deaton and Laroque, 1992). Accordingly, an implication of our analysis is that most of the long-lived shocks are likely to be downward movements in prices, which have important policy consequences for domestic and international schemes to stabilize earnings from commodity exports.

\section{Implications of Persistence Measures for Stabilization Schemes}

What should be the policy response (if any) of commodity-exporting developing countries to shocks to world commodity prices? If the persistence of such shocks is relatively short-lived, then scope does exist for domestic and international stabilization schemes to dampen the effects of price shocks, and for external borrowing and compensatory finance to smooth the path of national income and consumption. In such circumstances, it is less likely that the financing, storage, and output-reduction costs of stabilization schemes will exceed any consumption-smoothing benefits derived from the stability of export returns. However, if shocks to the price of any given country's commodity of interest are demonstrated to be long-lived, then the cost of operating a stabilization scheme will most likely exceed any associated smoothing benefits. In this situation there is likely to be little scope for domestic or international stabilization schemes to be successfully implemented. ${ }^{24}$

\footnotetext{
${ }^{23}$ Long lead times in the production of crude petroleum (particularly for non-OPEC producers) can result in extended periods of excess productive capacity and flat prices. In contrast, the lead times for the production of refined fuels (heating oil and gasoline) are relatively short.

${ }^{24}$ Aside from how long commodity price shocks are expected to last, the sustainability of commodity stabilization schemes will be dependent on the stabilization rule adopted and, as discussed above, the size and frequency of shocks.
} 


\section{Domestic stabilization schemes}

Domestic price stabilization schemes, typically taking the form of either buffer stock schemes or those that operate a buffer fund, are often implemented in commodity-exporting countries to reduce the effects of volatile world commodity prices on domestic prices or export returns. Attempts by government to stabilize prices to smooth the incomes of producers will most likely involve such stabilization funds holding a large amount of foreign reserves, or having the capacity to borrow large amounts of external capital, if there is a slow process of meanreversion following (adverse) shocks to commodity prices. The opportunity cost of such reserves is likely to be large, given that the rate of return from investment in many capital-deficient developing countries is likely to exceed the return on foreign reserve holdings (Deaton, 1992; and Borensztein and others, 1994). In addition, the accumulation of reserves during long-lasting price booms would be expensive (in terms of forgone earnings), most likely politically difficult to maintain, and prone to be used to fund unproductive investments (Deaton, 1992).

Many national commodity stabilization schemes, typically involving countries with market power in the determination of world prices for particular commodities, were terminated during the 1980s and 1990s owing to their financial unsustainability. A prominent example of the demise of a national stabilization arrangement concerns the collapse of the Australian wool stabilization scheme in 1992 (Bardsley, 1994). Our findings for wool indicate that while shocks to coarse wool and fine wool both have finite persistence (median HLS of 70 and 57 months, respectively), their associated 90 percent confidence intervals include infinity. Accordingly, while half the actual realizations of the HLS for both types of wool will be less than the median HLS (and so potentially financeable under any buffer stock arrangement), half will exceed the median, and 5 percent (or more) of the realizations will be permanent. Accordingly, both types of wool do not appear to be likely candidates for the successful operation of any price stabilization arrangement.

\section{International commodity agreements}

Many international commodity agreements designed to stabilize world commodity prices and raise returns to commodity producers (typically through buffer stock operations or export controls) also failed in the 1980s and 1990s, as the cost of maintaining them in the face of persistent price falls became unsustainable. International commodity agreements, typically using buffer stocks or export controls as tools of market intervention, were terminated for the following commodities: International Sugar Agreement (commenced in 1954, lapsed in 1984 owing to the expiry of export quotas); International Tin Agreement (commenced in 1954, collapsed in 1985 owing to the exhaustion of the resources of buffer stock scheme); International Cocoa Agreement (commenced in 1972, suspended in 1988 owing to an inability to finance the buffer stock scheme); and the International Coffee Agreement (commenced in 1962, suspended in 1989 after export quotas were allowed to expire). Only the International Natural Rubber Agreement 
(commenced in 1980) continues to give rise to active market intervention, although its role is being increasingly questioned by major producing countries.

While most international commodity agreements either lapsed or collapsed in the $1980 \mathrm{~s}$, the continuing relative decline and high variability of primary commodity prices in the 1990s may provide the catalyst for associations of producer countries to increasingly attempt to take unilateral action (as in the case of coffee in 1993-94) to manage supply in order to raise world prices (Gilbert, 1996). Our findings indicate that for those commodities, which experience very slow reversion in prices to their mean, of which coffee is a prime example, the temptation to take such action should be resisted, as the long-lived nature of shocks to coffee prices makes such schemes likely to be unsustainable (Table 2 and Figures 5d-5e).

International commodity agreements and their national counterparts (particularly domestic stabilization funds) have all suffered from a predilection to stabilize prices at an excessively high level, either because of a failure to properly account for deterministic trends or in an effort to subsidize producer returns. ${ }^{25}$ For a buffer-fund-based commodity agreement to remain viable, support prices need to be flexible downward when the current level proves to be unsustainable; in practice, such a price movement often becomes politically impossible, leading to the demise of such arrangements.

Not coincidentally, many of these failed international stabilization schemes involved commodities, which our analysis demonstrates exhibit long-lived shocks-cocoa, coffee, and sugar (see Tables 2-3 and Figures 5d-5e). In contrast, our analysis finds that rubber, which has the one surviving international commodity agreement, experiences relatively short-lived shocks (see Tables 2-3 and Figure 5b). Our findings also indicate that the commodity associated with the most famous producer cartel, petroleum, also experiences highly persistent shocks (see Tables 2-3 and Figure 5e). While the association of petroleum producers survives, OPEC's membership has not been broad enough to enable it to influence prices without subsequent loss of market share. ${ }^{26}$

\section{Compensatory finance}

An important example of compensatory financing is the IMF's CCFF. The CCFF, which began in 1988, was preceded by the Compensatory Financing Facility (CFF), which began in 1963. The CCFF is designed to smooth the effects of a temporary, exogenously caused shortfall in merchandise export receipts in a particular country below its medium-term trend. It recognizes the distinction

\footnotetext{
${ }^{25}$ See Gilbert (1996) for a description of these international agreements, and for the tendency of these ostensibly price-volatility-reducing schemes to evolve into price-raising schemes.

${ }^{26}$ One interpretation of the results presented in Table 3 and Figure 5 is that the persistence of shocks in regulated markets (such as sugar (EU)) differs from those in unregulated markets (such as sugar in the free market), owing to the influence of the market intervention measures. However, an alternative interpretation is that commodities with short-lived shocks provide scope for successful market intervention. This can be contrasted with the failed interventions in markets for commodities with long-lived shocks, as detailed in this section (see Tables 2-3 and Figures $5 \mathrm{~d}-5 \mathrm{e}$ ).
} 
between short-lived and long-lived shocks, and that the latter should not be financed but involve structural adjustment of the economy to its new long-run level of national income and consumption (Kaibni, 1986). ${ }^{27}$ The CCFF seeks to provide countries lacking either sufficient reserves, or the capacity to borrow externally, with the capacity to smooth the path of national consumption in the presence of a temporary shock to export earnings. Use of the CCFF by developing countries peaked at 48 percent of credit extended by the IMF in 1978. While drawings under the CCFF were active in the 1980s and early 1990s, drawings after 1993 were minimal until 1998, when use of the CCFF jumped sharply to comprise over 10 percent of IMF credit. ${ }^{28}$

The long-lived nature of shocks to many commodity prices indicates that in these highly persistent cases the scope for commodity stabilization policy is limited. Similarly, income-stabilizing schemes providing compensatory finance (such as the IMF's CCFF) are predicated on the assumption that the temporary shortfall in export earnings will be self-reversing. This assumption will not hold true (on average) where the shortfall in export earnings is (for given supply) caused by an adverse price shock to commodities that are subject to long-lived price shocks. ${ }^{29}$ Of course, on average this assumption may hold true where the shortfall in export earnings is caused by either (for given supply) an adverse price shock to commodities subject to short-lived price shocks or (for given price) a temporary fall in supply.

The resurgence of lending by the IMF under the CCFF in the late-1990s has largely involved countries experiencing adverse supply shocks to important commodity exports. For example, both Pakistan (December 1998) and Azerbaijan (January 1999) have received funds under the CCFF for temporary shortfalls in export earnings arising from weather-induced adverse shocks to their harvests of cotton, an important exportable (see Table 1). While the temporary nature of these supply-based shocks to export earnings is clear, our empirical findings indicate that the case for a CCFF to provide compensatory finance for any shortfall in cotton export earnings arising from an adverse shock to cotton prices (given supply) would be much weaker. While cotton has price shocks of finite persistence, they are typically very long-lived (median HLS of 151 months), and its associated 90 percent confidence interval includes infinity

${ }^{27}$ The CFF was extended in 1979 to include temporary shortfalls in tourism receipts and workers' remittances, and in 1981 to include temporary increases in expenditure on imports of foodstuffs (in particular, cereals). Countries drawing on the CCFF are required to repay their loans in equal quarterly installments, beginning 39 months and ending 60 months after the date of disbursement. The rate of charge is the same as that applying to the use of the IMF's general financial resources.

${ }^{28}$ Annual drawings under the CCFF averaged just under one-fifth (17.5 percent) of total credit (total purchases) extended by the IMF over the period 1963-98; by decade the share was 8 percent (1963-70), 26 percent (1971-80), 21 percent (1981-90), and 11 percent (1991-98). The peak years for drawings under the CCFF by decade, largely coinciding with troughs in world commodity prices were: 1967 (35 percent of Fund credit); 1978 (48 percent); 1982 (35 percent); and 1991 (40 percent).

${ }^{29}$ Strictly speaking, the CCFF is not a commodity-specific facility, but relates to the shortfall in total export earnings (Kaibni, 1986). In practice, the dependence of many developing countries on one or a few dominant commodity exports often makes this distinction a fine one (see Table 1 for the shares of dominant exports by commodity and country). 
(Tables 2-3 and Figure 5d). Although half the actual realizations of the HLS for cotton will be less than the median HLS (and so potentially temporary in nature), half will exceed the median HLS, and 5 percent (or more) of the realizations will be permanent. Accordingly, cotton does not appear (on average) to be a commodity that experiences temporary price shocks.

\section{Conclusion}

Movements in commodity prices are a key determinant of the performance of the world economy. They affect the level and stability of export incomes earned by developing countries, the cost of inputs to production in industrial countries, the allocation (sectoral and spatial) of world capital flows, and in particular rates of national economic growth. Knowledge of the persistence of shocks to commodity prices is an important input into the design of stabilization schemes to ameliorate the real macroeconomic effects of such shocks, particularly in developing countries.

In comparison with results from standard unit root regressions, which are largely uninformative on the question of the persistence of shocks, the scalar persistence measures used in this paper allow for a determination of the typical duration of price shocks as well as the variability of this duration. Using monthly International Monetary Fund data on 60 commodity price series over the period 1957-98, this study finds that shocks to the prices of many primary commodities are typically long-lasting, and that the variability of the persistence of price shocks is quite wide. Consequently, it is incorrect to view shocks to commodity prices as generally being temporary phenomena that largely reflect short-lived variability in supply interacting with relatively unchanging demand. Notwithstanding this, the persistence of shocks to commodity prices does vary greatly across commodities, with tree crops and metals typically having long-lasting shocks, and softwoods typically having short-lived shocks.

If shocks to commodity price series are extremely persistent, then an adverse price shock to any given commodity is likely to engender depressed prices for a long period of time. In such circumstances, government-supported price-stabilization activities and compensatory financing are likely to be ineffective, and external borrowing for consumption-smoothing is likely to be unsustainable. Even where shocks to commodity price series are relatively less persistent, the likelihood that the benefits of smoothing the path of domestic prices (given world commodity prices) exceed the cost of operating stabilization schemes or servicing external borrowing remains open to question. Moreover, while our results do not entirely rule out the successful operation of stabilization arrangements for many commodities, they do highlight the potential risk that such schemes will confront shocks, which last longer than typically observed, and thus may not be financially sustainable. 


\section{Appendix I}

\section{Specifications for Commodity Prices}

\begin{tabular}{|c|c|c|c|}
\hline Commodities & $\begin{array}{l}\text { World } \\
\text { Export } \\
\text { Weights } \\
\text { 1987-89 }\end{array}$ & Price Specifications & Unit \\
\hline \multicolumn{4}{|l|}{$\begin{array}{l}\text { Nonfuel } \\
\text { commodities }\end{array}$} \\
\hline Food & 32.9 & & \\
\hline Cereals & 13.6 & & \\
\hline Wheat & 7.4 & $\begin{array}{l}\text { U.S. No. } 1 \text { hard red winter, ordinary protein, prompt shipment f.o.b. } \\
\text { Gulf of Mexico ports (USDA, Grain and Feed Market News, Wash- } \\
\text { ington, D.C.). }{ }^{1}\end{array}$ & $\$ / M t$ \\
\hline Maize & 4.1 & $\begin{array}{l}\text { U.S. No. } 2 \text { yellow, prompt shipment, f.o.b. Gulf of Mexico ports } \\
\text { (USDA, Grain and Feed Market News, Washington, D.C.). }{ }^{1}\end{array}$ & $\$ / \mathrm{Mt}$ \\
\hline Rice & 2.1 & $\begin{array}{l}\text { Thai, white milled, } 5 \text { percent broken, nominal price quotes, f.o.b. } \\
\text { Bangkok (USDA, Rice Market News, Little Rock, Arkansas). }{ }^{2}\end{array}$ & $\$ / M t$ \\
\hline \multicolumn{4}{|c|}{$\begin{array}{l}\text { Vegetable oils and } \\
\text { protein meals } \quad \mathbf{1 0 . 6}\end{array}$} \\
\hline Soybeans & 4.1 & U.S., c.i.f. Rotterdam (Oil World, Hamburg). ${ }^{2}$ & $\$ / \mathrm{Mt}$ \\
\hline Soybean meal & 12.8 & Arg., 45/46 percent protein, c.i.f. Rotterdam (Oil World, Hamburg). & $\$ / \mathrm{Mt}$ \\
\hline Soybean oil & 1.2 & $\begin{array}{l}\text { Dutch, f.o.b. ex-mill (Oil World, Hamburg). Prior to April 1973, } \\
\text { Dutch crude oil, ex-mill. }{ }^{2}\end{array}$ & $\$ / M t$ \\
\hline Palm oil & 1.2 & $\begin{array}{l}\text { Malaysian/Indonesian, c.i.f. Northwest European ports (Oil World, } \\
\text { Hamburg). Prior to } 1974 \text {, UNCTAD. }{ }^{2}\end{array}$ & $\$ / \mathrm{Mt}$ \\
\hline Coconut oil & 0.3 & Philippine/Indonesian, bulk, c.i.f. Rotterdam (Oil World, Hamburg). & $\$ / \mathrm{Mt}$ \\
\hline Fishmeal & 0.9 & $\begin{array}{l}\text { Any origin, } 64-65 \text { percent protein, c.i.f. Hamburg (Oil World, } \\
\text { Hamburg). Prior to 1964, Peruvian meal, (FAO Estimate). }{ }^{2}\end{array}$ & $\$ / M t$ \\
\hline $\begin{array}{l}\text { Groundnut } \\
\text { oil }\end{array}$ & 0.1 & $\begin{array}{l}\text { Any origin, c.i.f. Rotterdam (Oil World, Hamburg). Prior to } 1974 \text {, } \\
\text { Nigerian bulk, c. f. U.K. ports. }{ }^{2}\end{array}$ & $\$ / \mathrm{Mt}$ \\
\hline Meat & 5.2 & & \\
\hline Beef & 4.5 & $\begin{array}{l}\text { Australian and New Zealand, frozen boneless, } 85 \text { percent visible } \\
\text { lean cow meat, U.S. import price f.o.b. port of entry. Prior to } \\
\text { December 1975, } 90 \text { percent visible lean meat (The Yellow Sheet, } \\
\text { Urner Barry Publications, Chicago, Illinois). }{ }^{1}\end{array}$ & $\mathrm{Cts} / \mathrm{lb}$ \\
\hline Lamb & 0.7 & $\begin{array}{l}\text { New Zealand, PL, frozen, wholesale price at Smithfield Market, } \\
\text { London (New Zealand Meat Producers Board, Washington, D.C.; } \\
\text { prior to October 1985, The Financial Times, London). Prior to } \\
\text { October 1976, New Zealand D's (The Financial Times, London). }{ }^{2} \\
\text { From Oct. 1996, New Zealand, UK wholesale price, medium fat } \\
\text { content (The National Business Review). }\end{array}$ & $\mathrm{Cts} / \mathrm{lb}$ \\
\hline Sugar & 2.5 & & \\
\hline Free market & 1.7 & $\begin{array}{l}\text { International Sugar Organization price. Average of The New York } \\
\text { contract No. } 11 \text { spot price, and the London daily price, f.o.b. } \\
\text { Caribbean ports (International Sugar Organization, London and } \\
\text { The Journal of Commerce, New York). Prior to 1976, New York } \\
\text { contract No.11, spot price, f.o.b. Caribbean and Brazilian ports. }{ }^{1}\end{array}$ & $\mathrm{Cts} / \mathrm{lb}$ \\
\hline
\end{tabular}




\section{Specifications for Commodity Prices (continued)}

\begin{tabular}{|c|c|c|c|}
\hline Commodities & $\begin{array}{l}\text { World } \\
\text { Export } \\
\text { Weights } \\
1987-89\end{array}$ & Price Specifications & Unit \\
\hline United States & 0.2 & $\begin{array}{l}\text { CSCE contract No. 14, nearest futures position, c.i.f. New York, } \\
\text { (Wall Street Journal and Dow Jones). Prior to June 1985, U.S. } \\
\text { spot import price, contract No. 12, c.i.f. New York (Journal of } \\
\text { Commerce, New York and Weekly Review of the market, Coffee } \\
\text { Sugar and Cocoa Exchange, Inc., New York). }{ }^{1}\end{array}$ & $\mathrm{Cts} / \mathrm{lb}$ \\
\hline $\begin{array}{l}\text { European } \\
\text { Union }\end{array}$ & 0.6 & $\begin{array}{l}\text { EU import price, unpacked sugar, c.i.f. European ports. Negotiated } \\
\text { price for sugar from ACP countries to EU under the Sugar Protocol. } \\
\text { (EU Office in Washington D.C.). }{ }^{1}\end{array}$ & $\mathrm{Cts} / \mathrm{lb}$ \\
\hline Bananas & 1 & $\begin{array}{l}\text { Central American and Ecuador, first class quality tropical pack, } \\
\text { Chiquita, U.S. importer's price, f.o.r. U.S. ports (Direccion Ejecu- } \\
\text { tiva de la Union de Paises Exportadores de Banano, FAX UPEB, } \\
\text { Panama, Panama). Beginning January 1997, prices were estimated } \\
\text { based on the average wholesale price at New York City and } \\
\text { Chicago. Up to December 1986, U.S. Bureau of Labor Statistics. }{ }^{2}\end{array}$ & $\$ / 401 b$ \\
\hline Beverages & 6.8 & & \\
\hline Coffee & 4.2 & & \\
\hline Other milds & 3.1 & $\begin{array}{l}\text { International Coffee Organization (New York) price. Average of El } \\
\text { Salvador central standard, Guatemala prime washed and Mexico } \\
\text { prime washed, prompt shipment, ex-dock New York (Bloomberg } \\
\text { Business News). }{ }^{1}\end{array}$ & $\mathrm{Cts} / \mathrm{lb}$ \\
\hline Robusta & 1.1 & $\begin{array}{l}\text { International Coffee Organization (New York) price. Average of } \\
\text { Cote d'Ivoire Grade II and Uganda standard, prompt shipment, } \\
\text { ex-dock New York (Bloomberg Business News). Prior to July 1982, } \\
\text { arithmetic average of Angolan Ambriz } 2 \text { AA and Ugandan Native } \\
\text { Standard, ex-dock New York. }\end{array}$ & $\mathrm{Cts} / \mathrm{lb}$ \\
\hline Cocoa beans & 1.4 & $\begin{array}{l}\text { International Cocoa Organization daily price. Average of the three } \\
\text { nearest active futures trading months in the New York Cocoa Ex- } \\
\text { change at noon and the London Terminal market at closing time, } \\
\text { c.i.f. U.S. and European ports (The Financial Times, London). }{ }^{1}\end{array}$ & $\$ / \mathrm{Mt}$ \\
\hline Tea & 1.2 & $\begin{array}{l}\text { From July 1998, Mombasa auction price, all teas (International Tea } \\
\text { Committee, London) Prior to July } 1998 \text { is London auctions, average } \\
\text { price received for good medium, c.i.f., U.K. warehouses (The Tea } \\
\text { Brokers Association, The Financial Times, London). }{ }^{2}\end{array}$ & $\mathrm{Cts} / \mathrm{Kg}$ \\
\hline $\begin{array}{l}\text { Agricultural raw } \\
\text { materials }\end{array}$ & 32.3 & & \\
\hline Timber & 15.5 & & \\
\hline Hardwood & 5.4 & & \\
\hline Logs & 1.9 & $\begin{array}{l}\text { Malaysian, meranti, Sarawak best quality, sale price charged by im- } \\
\text { porters, Japan (World Bank, Washington, D.C.). From January } 1988 \\
\text { to February 1993, average of Sabah and Sarawak in Tokyo weight } \\
\text { by their respective import volumes in Japan. From February } 1993 \text { to } \\
\text { present, Sarawak only. }{ }^{3}\end{array}$ & $\$ / \mathrm{Cm}$ \\
\hline Sawnwood & d 3.5 & $\begin{array}{l}\text { Malaysian sawnwood, dark red meranti, select and better quality, } \\
\text { standard density, C\&F U.K. Port (Tropical Timbers, Surrey, England). }{ }^{3}\end{array}$ & $\$ / \mathrm{Cm}$ \\
\hline
\end{tabular}




\section{Specifications for Commodity Prices (continued)}

\begin{tabular}{|c|c|c|c|}
\hline Commodities & $\begin{array}{l}\text { World } \\
\text { Export } \\
\text { Weights } \\
1987-89\end{array}$ & Price Specifications & Unit \\
\hline Softwood & 10.1 & & \\
\hline Logs & 1.8 & $\begin{array}{l}\text { Average export price of Douglas-fir, Western hemlock and other } \\
\text { softwoods exported from Washington, Oregon, Northern California } \\
\text { and Alaska. (Pacific Northwest Research Station, USDA Forest Ser- } \\
\text { vice, Portland, OR). }{ }^{3}\end{array}$ & $\$ / \mathrm{Cm}$ \\
\hline Sawnwood & d 8.3 & $\begin{array}{l}\text { Average export price of Douglas-fir, Western hemlock and other } \\
\text { sawn softwood exported from Washington, Oregon, other Northern } \\
\text { California and Alaska. (Pacific Northwest Research Station, USDA } \\
\text { Forest Service, Portland, OR). }{ }^{2}\end{array}$ & $\$ / \mathrm{Cm}$ \\
\hline Cotton & 3.9 & $\begin{array}{l}\text { Middling 1-3/32 inch staple, Liverpool Index "A", average of the } \\
\text { cheapest five of fourteen styles, c.i.f. Liverpool (Cotton Outlook, } \\
\text { Liverpool). From January } 1968 \text { to May } 1981 \text { strict middling 1-1/16 } \\
\text { inch staple. Prior to 1968, Mexican 1-1/16. }\end{array}$ & $\mathrm{Cts} / \mathrm{lb}$ \\
\hline Wool & 3.8 & & \\
\hline Fine & 2.4 & $\begin{array}{l}\text { 64's clean, dry combed basis. (Commonwealth Secretariat, London, } \\
\text { England). }{ }^{2}\end{array}$ & $\mathrm{Cts} / \mathrm{Kg}$ \\
\hline Coarse & 1.4 & $\begin{array}{l}48 \text { 's clean, dry combed basis. Prior to January 1987, 50's. (Com- } \\
\text { monwealth Secretariat, London, England) }{ }^{2}\end{array}$ & $\mathrm{Cts} / \mathrm{Kg}$ \\
\hline Rubber & 3.3 & $\begin{array}{l}\text { Malaysian, No. } 1 \text { RSS, prompt shipment, f.o.b. Malaysian/ } \\
\text { Singapore ports (The Financial Times, London). }{ }^{1}\end{array}$ & $\mathrm{Cts} / \mathrm{lb}$ \\
\hline Tobacco & 2.2 & $\begin{array}{l}\text { U.S. import unit value of general unmanufactured tobacco. (USDA, } \\
\text { Foreign Agricultural Service). }\end{array}$ & $\$ / \mathrm{Mt}$ \\
\hline Hides & 3.6 & $\begin{array}{l}\text { U.S., Chicago packer's heavy native steers, over } 53 \text { lbs., wholesale } \\
\text { dealer's price, (formerly over } 58 \text { lbs.), f.o.b. shipping point (Wall } \\
\text { Street Journal, New York). Prior to November 1985, U.S. Bureau } \\
\text { of Labor Statistics, Washington, D.C. }{ }^{1}\end{array}$ & $\mathrm{Cts} / \mathrm{lb}$ \\
\hline Metals & 26.7 & & \\
\hline Copper & 6.4 & $\begin{array}{l}\text { London Metal Exchange, grade A cathodes, spot price, c.i.f. Euro- } \\
\text { pean ports (Wall Street Journal, New York and Metals Week, New } \\
\text { York). Prior to July 1986, higher grade, wirebars, or cathodes. }{ }^{1}\end{array}$ & $\mathrm{Cts} / \mathrm{lb}$ \\
\hline Aluminum & 10.2 & $\begin{array}{l}\text { London Metal Exchange, standard grade, spot price, minimum } \\
\text { purity } 99.5 \text { percent, c.i.f. U.K. ports (Wall Street Journal, New } \\
\text { York and Metals Week, New York). Prior to 1979, U.K. New pro- } \\
\text { ducer price, minimum purity } 99 \text { percent. }{ }^{1}\end{array}$ & $\$ / M t$ \\
\hline Iron ore & 3.7 & $\begin{array}{l}\text { Brazilian, Itabira standard sinterfeed, } 64.3 \text { percent iron, contract } \\
\text { price to Germany, f.o.b. Tubarao (Companhia Vale contract do } \\
\text { Rio Doce, Rio de Janeiro, Brazil ). }{ }^{4}\end{array}$ & $\begin{array}{l}\text { Cts/ } \\
\text { DMTU }\end{array}$ \\
\hline Tin & 1.1 & $\begin{array}{l}\text { London Metal Exchange, standard grade, spot price, c.i.f. Euro- } \\
\text { pean ports (Wall Street Journal, New York, New York). From Dec. } \\
1985 \text { to June } 1989 \text { Malaysian, straits, minimum From Dec. } 99 . \\
85 \text { percent purity, Kuala Lumpur Tin Market settlement price. Prior } \\
\text { to November 1985, London Metal Exchange (Wall Street Journal, } \\
\text { New York and Metals Week, New York). }{ }^{1}\end{array}$ & $\mathrm{Cts} / \mathrm{lb}$ \\
\hline
\end{tabular}




\section{Specifications for Commodity Prices (concluded)}

\begin{tabular}{|c|c|c|c|}
\hline Commodities & $\begin{array}{l}\text { World } \\
\text { Export } \\
\text { Weights } \\
1987-89\end{array}$ & Price Specifications & Unit \\
\hline Nickel & 1.6 & $\begin{array}{l}\text { London Metal Exchange, melting grade, spot price, c.i.f. Northern } \\
\text { European ports (Wall Street Journal, New York and Metals Week, } \\
\text { New York). Prior to } 1980 \text { INCO, melting grade, c.i.f. Far East and } \\
\text { American ports (Metal Bulletin, London). }{ }^{1}\end{array}$ & $\$ / \mathrm{Mt}$ \\
\hline Zinc & 2.8 & $\begin{array}{l}\text { London Metal Exchange, high grade } 98 \text { percent pure, spot price, } \\
\text { c.i.f. U.K. ports (Wall Street Journal and Metals Week, New York). } \\
\text { Prior to January 1987, standard grade. }{ }^{1}\end{array}$ & $\$ / \mathrm{Mt}$ \\
\hline Lead & 0.9 & $\begin{array}{l}\text { London Metal Exchange, } 99.97 \text { percent pure, spot price, c.i.f. } \\
\text { European ports (Wall Street Journal, New York and Metals Week, } \\
\text { New York ). }{ }^{1}\end{array}$ & $\$ / \mathrm{Mt}$ \\
\hline Fertilizers & 1.3 & & \\
\hline Phosphate rock & 0.7 & $\begin{array}{l}\text { Moroccan, } 70 \text { percent BPL, contract, f.a.s. Casablanca (The World } \\
\text { Bank, Washington). Prior to 1981, } 72 \text { percent BPL, World f.a.s. } \\
\text { Casablanca. }{ }^{2}\end{array}$ & $\$ / \mathrm{Mt}$ \\
\hline $\begin{array}{l}\text { TSP (triple } \\
\text { super-phosphate }\end{array}$ & e) 0.6 & $\begin{array}{l}\text { U.S. bulk, spot price, f.o.b. Gulf of Mexico ports, (Fertilizer } \\
\text { Week, CRU International Ltd., London, England). }{ }^{1}\end{array}$ & $\$ / \mathrm{Mt}$ \\
\hline \multicolumn{4}{|l|}{ Petroleum } \\
\hline Spot Crude & & $\begin{array}{l}\text { U.K. light, Brent Blend 38o API, spot price, f.o.b. U.K. ports } \\
\text { (Bloomberg Business News). Prior to 1984, North African Light } \\
\text { 37/44o API (Petroleum Intelligence Weekly, New York). Prior to } \\
\text { 1974, Libyan Brega 40o API, posted price, ex Marsa El Brega } \\
\text { (Platt's Oil Price Handbook and Almanac, New York). Prior to 1961, } \\
\text { Qatar Um Said 39o API, posted price, f.o.b. (Platt's Oil Price Hand- } \\
\text { Handbook and Almanac, New York). }{ }^{1}\end{array}$ & $\$ / b b l$ \\
\hline Gasoline & & $\begin{array}{l}\text { U.S. Gulf, regular unleaded, Petroleum Product Assessments } \\
\text { (Reuter's News Services). }{ }^{1}\end{array}$ & Cts/gal \\
\hline Natural Gas & & $\begin{array}{l}\text { Russian border price in Germany (World Gas Intelligence, } \\
\text { New York). }\end{array}$ & $\$ / 000 \mathrm{~m}^{3}$ \\
\hline Heating Oil & & No. 2 heating oil, NYMEX, (Reuter's News Services). ${ }^{1}$ & Cts/gal \\
\hline Gold & & UK 99.5 percent Fine, PM Fixing, Average daily. & \$/ounce \\
\hline MUV & & $\begin{array}{l}\text { Unit value index (in U.S. dollars) of manufactures exported by } \\
20 \text { developed countries, with country weights based on the coun- } \\
\text { tries' total } 1990 \text { exports of manufactures (that is, } 1990=100 \text { ). }\end{array}$ & \\
\hline
\end{tabular}

Source: International Monetary Fund.

${ }^{1}$ Average of daily quotations.

${ }^{2}$ Average of weekly quotations.

${ }^{3}$ Monthly quotations

${ }^{4}$ The price is quoted in cents per 1 percent Fe dry metric ton f.o.b. (DMTU). For 64.3 percent Itabira fines, a price of $28.6 \mathrm{cts} / \mathrm{DMTU}$ is equal to US\$18.38 per metric ton. 


\section{APPENDIX II}

\section{Persistence of Commodity Price Shocks Across Exchange Rate Regimes}

To assume that the time series properties of commodity prices are unchanged over time is potentially restrictive, especially given the nature of the shocks to the world economy during the sample period examined here. Cuddington and Liang (1997) show that the volatility of commodity prices increased significantly after the Bretton Woods system broke down. ${ }^{30} \mathrm{We}$ examine whether the breakdown of the fixed exchange rate system in the 1970s also lead to a change in the cyclical behavior of the levels of real commodity prices.

From a theoretical point of view, a class of "equilibrium models" demonstrate neutrality of the nominal exchange arrangement for the behavior of real macroeconomic variables such as real exchange rates (Helpman, 1981). On the other hand, the observed relatively slow adjustment of goods prices compared with those of asset prices, such as exchange rates and commodity prices, suggests that nominal exchange rate movements may have an impact on real economic variables (Mussa, 1986). Although both primary commodities and manufactures are internationally traded, their underlying market structures have long been perceived to be different. While the prices of most non-oil primary commodities are largely determined in wellorganized auction markets, the markets for many manufactured goods are thought to be much less competitive. Consequently, how fast changes in the exchange rate pass through to the prices of final goods may be quite different for the two goods (Liang, 1998). Therefore, it is conceivable that the differing speeds of exchange rate pass-through may lengthen the persistence of price shocks in the presence of flexibility in the nominal exchange rate. If we assume that the processes generating real shocks to commodity prices (from either the supply or demand sides) are not exchange rate regime-dependent, the degree of shock persistence may increase under a flexible exchange regime if manufactured goods prices respond more slowly to nominal exchange rate changes than do the prices of primary commodities.

To examine this contention empirically, this Appendix compares the persistence of real commodity price shocks across nominal exchange rate regimes for those commodities with monthly data for the full sample (January 1957 to December 1998), and follows Cuddington and Liang (1997) in denoting the fixed exchange rate regime as January 1957-July 1971, and the flexible exchange rate regime as August 1971-December 1998.

For the 51 commodity price series with observations in both exchange rate regimes, in 23 series the median-unbiased calculation of the HLS (derived from equation (4)) is less than permanent in only one of the two regimes; for another 15 series the HLS is permanent (infinitely persistent) in both regimes; and for 13 series the HLS is permanent (infinitely persistent) in neither regime. ${ }^{31}$ Of the 36 cases with HLS less than infinity in at least one of the two regimes, in 17 of the cases the persistence is lower in the fixed exchange rate regime, and in 10 of these 17 cases the difference in HLS is statistically significant (as the confidence interval of the HLS for the fixed exchange rate regime does not contain the HLS for that same commodity

30Using a univariate GACH process to model the six main categories of commodities over the period of 1957-96, Cuddington and Liang (1997) show that change in the nominal exchange regime leads to parameter shifts in the GARCH process, and hence in the degree to which shocks to commodity price volatility persist over time.

${ }^{31}$ The 9 commodity series excluded from this analysis, due to the absence of observations in the fixed exchange rate regimes are gasoline, hardwood, heating oil, softwood $(\operatorname{logs})$, hardwood $(\operatorname{logs})$, natural gas, hardwood (sawnwood), softwood (sawnwood), and softwood. 


\section{Table 4. Effect of Nominal Exchange Rate Regime on the Median HLS for Commodities, 1957:1-1998:12}

$\begin{array}{lcc}\text { Commodity } & \begin{array}{c}\text { Fixed Period (1957:1-1971:7): } \\ \text { Number of Month for Median HLS }\end{array} & \begin{array}{c}\text { Flexible Period (1971:8-1988:12): } \\ \text { Number of Months for Median HLS }\end{array} \\ \text { All commodities } & 16.28 & \infty \\ \text { Bananas } & 1.09 & 1.81 \\ \text { Beef } & 3.50 & 43.43 \\ \text { Food } & 15.87 & \infty \\ \text { Lamb } & 3.80 & 15.63 \\ \text { Maize } & 13.32 & 63.41 \\ \text { Meat } & 3.58 & 44.58 \\ \text { Sugar (US) } & 3.87 & 36.35 \\ \text { Tea } & 2.17 & 20.99 \\ \text { Tobacco } & 6.96 & \infty \\ \text { Sugar (EU) } & \infty & 4.27 \\ \text { Wool (coarse) } & \infty & 25.02\end{array}$

Source: Authors' calculations, derived using equation (4) and the median-unbiased estimates of the autoregressive parameter $(\alpha)$.

Note: HLS is, for $\alpha \geq 0$, the length of time until the impulse response of a unit shock to an economic time series is half its initial magnitude (see equation (4)).

estimated for the flexible exchange rate regime). ${ }^{32}$ Conversely, in the 19 cases where the persistence is lower in the flexible exchange rate regime than in the fixed regime, in only two of the cases are the difference in HLS across exchange rate regimes statistically significant (as the confidence interval of the HLS for the flexible exchange rate regime contains the HLS for that same commodity estimated for the fixed exchange rate regime).

Table 4 reports the HLS for the above-mentioned 12 commodity price series that exhibit a significant difference in the persistence of shocks across exchange rate regimes. Shock persistence was higher in the fixed exchange rate period only for sugar (EU) and wool (coarse), both of which traditionally have their prices determined in markets subject to public intervention. On the other hand, for most of the market-determined prices for the eight individual commodities, shock persistence increased dramatically after the Bretton Woods fixed exchange rate arrangement collapsed in 1971. The HLS increased from about 15 months to infinity for both the all commodities and food indices.

In summary, unlike the conclusions reached by Cuddington and Liang (1997) regarding volatility, we find only weak support for the hypothesis that the nominal exchange rate regime has had a significant impact on the extent to which shocks to the level of commodity prices persist over time. Nevertheless, these findings still shed light on how we should analyze the interactions between the movements of nominal exchange rate and commodity prices.

There is a rich literature on the question of how dollar-denominated primary commodity prices respond to changes in the value of the dollar against other currencies (see Dornbusch, 1985). A large number of studies have found that dollar commodity prices have a greater than unit response to changes in the value of the dollar. ${ }^{33}$ On the other hand, most empirical studies

${ }^{32}$ The statistically significant price series are bananas, beef, lamb, maize, meat, sugar (U.S.), tea, tobacco (individual price series), and all commodities and food (aggregate price series).

${ }^{33}$ See Gilbert's (1990) Table 1 for a summary of estimates of the long-run exchange rate elasticity. 
on how responsive trade prices of manufactures are to exchange rate changes, especially in the U.S. economy, have found that exchange rate pass-though has been far from unity (Mann, 1986). The findings of this paper support the hypothesis that, at least for certain commodities, exchange pass-through mechanisms for primary commodities and manufactured goods are different. In addition, for those individual commodities that do exhibit significantly different patterns of shock persistence across exchange rate regimes, our findings suggest that it has most likely become more difficult for countries that specialize in these commodities to smooth the fluctuations in their export earnings in a world with greater exchange rate flexibility.

\section{APPENDIX III}

\section{Table 5. Monte Carlo Estimates of Quantiles of the Least Squares Estimator of $\alpha$ for Andrews' (1993) Model with Intercept and Time Trend, for 300,400 , and 500 Observations}

\begin{tabular}{crrrrrrrrrr} 
& \multicolumn{3}{c}{$T+1=300$} & \multicolumn{3}{c}{$T+1=400$} & \multicolumn{3}{c}{$T+1=500$} \\
$\alpha /$ Quantile & .05 & .5 & .95 & .05 & .5 & .95 & .05 & .5 & .95 \\
-.999 & -1.002 & -.998 & -.982 & -1.002 & -.998 & -.985 & -1.001 & -.998 & -.987 \\
-.80 & -.849 & -.798 & -.734 & -.844 & -.799 & -.745 & -.840 & -.799 & -.751 \\
-.60 & -.671 & -.600 & -.519 & -.622 & -.600 & -.531 & -.656 & -.600 & -.539 \\
-.40 & -.485 & -.402 & -.313 & -.475 & -.402 & -.324 & -.467 & -.401 & -.332 \\
-.20 & -.296 & -.204 & -.110 & -.283 & -.203 & -.121 & -.274 & -.202 & -.130 \\
.00 & -.101 & -.006 & .089 & -.086 & -.004 & .078 & -.078 & -.003 & .069 \\
.10 & -.003 & .093 & .187 & .012 & .095 & .176 & .021 & .096 & .168 \\
.20 & .096 & .192 & .284 & .111 & .194 & .273 & .122 & .195 & .266 \\
.30 & .197 & .291 & .380 & .211 & .293 & .370 & .223 & .295 & .363 \\
.40 & .298 & .390 & .475 & .313 & .393 & .465 & .324 & .394 & .459 \\
.50 & .399 & .489 & .568 & .416 & .492 & .560 & .427 & .494 & .555 \\
.60 & .503 & .588 & .660 & .520 & .591 & .654 & .530 & .593 & .649 \\
.70 & .609 & .687 & .751 & .620 & .690 & .746 & .635 & .692 & .742 \\
.80 & .717 & .785 & .839 & .732 & .789 & .835 & .741 & .792 & .833 \\
.85 & .772 & .834 & .881 & .787 & .839 & .879 & .795 & .841 & .877 \\
.90 & .827 & .883 & .923 & .841 & .888 & .921 & .850 & .890 & .921 \\
.93 & .861 & .912 & .946 & .875 & .917 & .946 & .884 & .920 & .946 \\
.97 & .906 & .950 & .975 & .920 & .955 & .976 & .929 & .959 & .977 \\
.99 & .923 & .966 & .988 & .941 & .972 & .989 & .951 & .976 & .990 \\
1.00 & .929 & .970 & .991 & .946 & .977 & .993 & .957 & .982 & .995 \\
& & & & & & & & &
\end{tabular}

Source: Authors' calculations.

Notes: The values of the median function $(m(\alpha))$ contained in the table were calculated using Monte Carlo simulations of 10,000 replications. 


\section{REFERENCES}

Andrews, Donald, 1993, "Exactly Median-Unbiased Estimation of First-Order Autoregressive/Unit Root Models," Econometrica, No. 61, pp. 139-65.

— Autoregressive Models," Journal of Business and Economic Statistics, No. 12, pp. 187-204.

Bardsley, Peter, 1994, "The Collapse of the Australian Wool Reserve Price Scheme," Economic Journal, Vol. 104, pp. 1087-1105.

Borensztein, Eduardo, Mohsin S. Khan, Carmen M. Reinhart, and Peter Wickham, 1994, The Behavior of Non-Oil Commodity Prices, IMF Occasional Paper 112 (Washington: International Monetary Fund).

_ a and Carmen M. Reinhart, 1994, "The Macroeconomic Determinants of Commodity Prices," IMF Staff Papers, No. 41, pp. 236-61 (Washington: International Monetary Fund).

Cashin, Paul, C. John McDermott, and Alasdair Scott, 1999, "Booms and Slumps in World Commodity Prices," IMF Working Paper 99/155 (Washington: International Monetary Fund).

Cochrane, John H., 1988, “How Big Is the Random Walk in GNP?" Journal of Political Economy, No. 96, pp. 893-920.

Cuddington, John T., and Hong Liang, 1997, "Commodity Price Volatility Across Exchange Rate Regimes," Working Paper, No. 97-17 (Washington: Georgetown University).

—, and Carlos M. Urzúa, 1989, "Trend and Cycles in the Net Barter Terms of Trade: A New Approach,” Economic Journal, No. 99, pp. 426-42.

DeJong, David N., John C. Nankervis, N.E. Savin, and Charles H. Whiteman, 1992, "Integration Versus Trend Stationarity in Time Series," Econometrica, No. 60, pp. 423-33.

Deaton, Angus S., 1992, "Commodity Prices, Stabilization, and Growth in Africa," Research Program in Development Studies, Discussion Paper 166 (New Jersey: Princeton University).

— , and Guy Laroque, 1992, "On the Behavior of Commodity Prices," The Review of Economic Studies, Vol. 59, pp. 1-24.

Dickey, David A., and Wayne A. Fuller, 1979, "Distribution of the Estimators for Autoregressive Time Series with a Unit Root," Journal of the American Statistical Association, No. 74, pp. 427-31.

Dornbusch, Rudiger, 1985, "Exchange Rates and Prices," NBER Working Paper, No. 1769 (Cambridge, Massachusetts: MIT Press).

Gersovitz, Mark and Christina H. Paxson, 1990, "The Economies of Africa and the Prices of Their Exports," Princeton Studies in International Finance, No. 68 (New Jersey: Princeton University).

Gilbert, Christopher L., 1990, "The Response of Primary Commodity Prices to Exchange Rate Changes," Department of Economics Working Paper, No. 208 (London: University of London).

1993, "Domestic Price Stabilization Schemes for Developing Countries," in Managing Commodity Price Risk in Developing Countries, ed. by S. Claessens and Ronald C. Duncan (Baltimore, Maryland: Johns Hopkins University Press; Washington: The World Bank), pp. 30-67.

— 1996, "International Commodity Agreements: An Obituary Notice," World Development, Vol. 24, pp. 1-19. 
Grilli, Enzo R. and Maw C. Yang, 1988, "Primary Commodity Prices, Manufactured Goods Prices, and Terms of Trade of Developing Countries: What the Long Run Shows," World Bank Economic Review, No. 2, pp. 1-48.

Helpman, Elhanan, 1981, "An Exploration in the Theory of Exchange-Rate Regimes," Journal of Political Economy, No. 89, pp. 865-90.

International Monetary Fund, 1986, Primary Commodities: Market Developments and Outlook (Washington: International Monetary Fund).

— 1999, International Financial Statistics (Washington: International Monetary Fund).

Kaibni, Nihad, 1986, "Evolution of the Compensatory Financing Facility," Finance and Development, Vol. 23, pp. 24-27 (Washington: International Monetary Fund).

León, Javier, and Raimundo Soto, 1997, "Structural Breaks and Long-Run Trends in Commodity Prices," Journal of International Development, Vol. 9, pp. 347-66.

Liang, Hong, 1998, "The Volatility of the Relative Price of Commodities in Terms of Manufactures Across Exchange Regimes: A Theoretical Model," IMF Working Paper 98/163 (Washington: International Monetary Fund).

McDermott, C. John, 1994, "Structural and Evolutionary Change in Econometric Models," PhD Dissertation, Department of Economics (New Haven: Yale University).

Mann, Catherine L., 1986, "Prices, Profit Margins, and Exchange Rates," Federal Reserve Bulletin, No. 72, pp. 366-79.

Mussa, Michael, 1986, "Nominal Exchange Rate Regimes and the Behavior of Real Exchange Rates: Evidence and Implications," Carnegie-Rochester Conference Series on Public Policy, No. 25, pp. 117-214.

Orcutt, Guy H., 1948, "A Study of the Autoregressive Nature of the Time Series Used for Tinbergens Model of the Economic System of the United States, 1891-1932," Journal of the Royal Statistical Society, Series B, No. 10, pp. 1-48.

Perron, Pierre, 1989, "The Great Crash, the Oil Price Shock, and the Unit Root Hypothesis," Econometrica, Vol. 57, pp. 1361-1401.

Phillips, Peter, and Pierre Perron, 1988, “Testing for a Unit Root in Time Series Regression," Biometrika, No. 75, pp. 335-46.

Prebisch, Raúl, 1950, The Economic Development of Latin America and Its Principal Problems (New York: United Nations).

Reinhart, Carmen, and Peter Wickham, 1994, "Commodity Prices: Cyclical Weakness or Secular Decline?," IMF Staff Papers, Vol. 41, pp. 175-213.

Rudebusch, Glenn D., 1992, "Trends and Random Walks in Macroeconomic Time Series: A Reexamination," International Economic Review, No. 33, pp. 661-80.

Singer, Hans W., 1950, "The Distribution of Gains Between Investing and Borrowing Countries, American Economic Review, Papers and Proceedings, Vol. 40, pp. 473-85.

Stock, James, 1991, "Confidence Intervals for the Largest Autoregressive Root in U.S. Macroeconomic Time Series," Journal of Monetary Economics, No. 28, pp. 435-59.

World Bank, 1994, Global Economic Prospects and the Developing Countries (Washington: World Bank).

Zivot, Eric, and Donald Andrews, 1992, "Further Evidence on the Great Crash, the Oil Price Shock and the Unit-Root Hypothesis," Journal of Business and Economic Statistics, Vol. 10, pp. 251-70. 